\title{
Non-LTE effects on the lead and thorium abundance determinations for cool stars
}

\author{
L. Mashonkina ${ }^{1,2}$, A. Ryabtsev ${ }^{3}$, and A. Frebel ${ }^{4,5}$ \\ 1 Universitäts-Sternwarte München, Scheinerstr. 1, 81679 München, Germany \\ e-mail: lyuda@usm.lmu.de \\ 2 Institute of Astronomy, Russian Academy of Sciences, 119017 Moscow, Russia \\ e-mail: lima@inasan.ru \\ 3 Institute of Spectroscopy, Russian Academy of Sciences, 142190 Troitsk, Moscow region, Russia \\ ${ }^{4}$ Massachussetts Institute of Technology, Kavli Institute for Astrophysics and Space Research, 77 Massachusetts Avenue, Cambridge, \\ MA 02139, USA \\ e-mail: afrebel@mit.edu \\ ${ }^{5}$ Harvard-Smithsonian Center for Astrophysics, 60 Garden St, Cambridge, MA 02138, USA
}

Received 9 January 2012 / Accepted 6 February 2012

\begin{abstract}
Context. Knowing accurate lead abundances of metal-poor stars provides constraints on the Pb production mechanisms in the early Galaxy. Accurately deriving thorium abundances permits a nucleo-chronometric age determination of the star.

Aims. We aim to improve the calculation of the $\mathrm{Pb}$ I and $\mathrm{Th}$ II lines in stellar atmospheres based on non-local thermodynamic equilibrium (non-LTE) line formation, and to evaluate the influence of departures from LTE on $\mathrm{Pb}$ and Th abundance determinations for a range of stellar parameters by varying the metallicity from the solar value down to $[\mathrm{Fe} / \mathrm{H}]=-3$.

Methods. We present comprehensive model atoms for $\mathrm{Pb}$ I and $\mathrm{Th}$ II and describe calculations of the $\mathrm{Pb}$ i energy levels and oscillator strengths.

Results. The main non-LTE mechanism for $\mathrm{Pb}_{\mathrm{I}}$ is the ultraviolet overionization. We find that non-LTE leads to systematically depleted total absorption in the $\mathrm{Pb}$ I lines and accordingly, positive abundance corrections. The departures from LTE increase with decreasing metallicity. Using the semi-empirical model atmosphere HM74, we determine the lead non-LTE abundance for the Sun to be $\log \varepsilon_{\mathrm{Pb}, \odot}=2.09$, in agreement with the meteoritic lead abundance. We revised the $\mathrm{Pb}$ and Eu abundances of the two strongly r-process enhanced stars CS 31082-001 and HE 1523-0901 and the metal-poor stellar sample. Our new results provide strong evidence of universal $\mathrm{Pb}$-to-Eu relative r-process yields during the course of Galactic evolution. The stars in the metallicity range $-2.3<[\mathrm{Fe} / \mathrm{H}]<-1.4$ have $\mathrm{Pb} / \mathrm{Eu}$ abundance ratios that are, on average, 0.51 dex higher than those of strongly r-process enhanced stars. We conclude that the s-process production of lead started as early as the time when Galactic metallicity had reached $[\mathrm{Fe} / \mathrm{H}]=-2.3$. The average $\mathrm{Pb} / \mathrm{Eu}$ abundance ratio of the mildly metal-poor stars, with $-1.4 \leq[\mathrm{Fe} / \mathrm{H}] \leq-0.59$, is very close to the corresponding Solar System value, in line with the theoretical predictions that $\mathrm{AGB}$ stars with $[\mathrm{Fe} / \mathrm{H}] \simeq-1$ provided the largest contribution to the solar abundance of s-nuclei of lead. The departures from LTE for Th in are caused by the pumping transitions from the levels with $E_{\text {exc }}<1 \mathrm{eV}$. Non-LTE leads to weakened Th II lines and positive abundance corrections. Overall, the abundance correction does not exceed 0.2 dex when collisions with $\mathrm{H}_{\mathrm{I}}$ atoms are taken into account in statistical equilibrium calculations.
\end{abstract}

Key words. line: formation - nuclear reactions, nucleosynthesis, abundances - Sun: abundances - stars: abundances stars: atmospheres

\section{Introduction}

Lead $(Z=82)$, thorium $(Z=90)$, and uranium $(Z=92)$ are the three heaviest elements observed in metal-poor (MP) stars, with $[\mathrm{Fe} / \mathrm{H}]^{1}<-1$. Only one detection of bismuth $(Z=83)$ has ever been made for an MP star (Ivans et al. 2005), because the only suitable line, Bi I $3067 \AA$, lies in a very crowded spectral region in the near-UV that is difficult to access. All nuclei between ${ }^{209} \mathrm{Bi}$ and ${ }^{244} \mathrm{Pu}$ that exist in nature, are radioactive. Among them, only Th and $U$ have long-lived isotopes with half-lives longer than $1 \mathrm{Gyr}$, namely $t_{1 / 2}\left({ }^{232} \mathrm{Th}\right) \simeq 14 \mathrm{Gyr}$ and $t_{1 / 2}\left({ }^{238} \mathrm{U}\right) \simeq 4.5 \mathrm{Gyr}$, and can be detected in the spectra of MP stars.

The knowledge of stellar $\mathrm{Pb}$ abundances provides a clearer understanding of the production mechanisms of lead during the

\footnotetext{
${ }^{1}$ In the classical notation, where $[\mathrm{X} / \mathrm{H}]=\log \left(N_{\mathrm{X}} / N_{\mathrm{H}}\right)_{\text {star }}-$ $\log \left(N_{\mathrm{X}} / N_{\mathrm{H}}\right)_{\text {Sun }}$.
}

evolution of the Galaxy. Lead is produced by two neutroncapture processes that have different timescales, i.e., in the slow (s) process occurring during the thermally pulsing asymptotic giant branch $(\mathrm{AGB})$ phase of intermediate-mass $\left(2-8 M_{\odot}\right)$ stars (see for example, Gallino et al. 1998) and the rapid (r) process. The astrophysical site at which the r-process operates has not yet been identified. Given that the heavy elements, such as $\mathrm{Ba}$ and $\mathrm{Eu}$, are detected in very metal-poor (old) stars, supernovae with progenitors of $8-10 M_{\odot}$ are the most promising candidates for r-process enrichment in the early Galaxy. We refer to, for example, the pioneering review of Hillebrandt (1978) and also Cowan \& Thielemann (2004) for further discussion of the r-process. Theoretical studies of the s-process have shown that in low-metallicity environment, heavy s-nuclei similar to those of $\mathrm{Pb}$ are more efficiently produced than lighter nuclei owing to both the larger ratio of free neutrons to Fe-peak seeds and 
an extended period of s-process nucleosynthesis (Gallino et al. 1998; Travaglio et al. 2001). It has been predicted that the AGB stars with $[\mathrm{Fe} / \mathrm{H}] \simeq-1$ made the greatest contribution to the solar abundance of s-nuclei of lead. According to Travaglio et al. (2001), the s-process fraction of the solar $\mathrm{Pb}$ amounts to $81 \%$.

The largest data set on lead abundances of MP stars was compiled by Roederer et al. (2010). They found that the stellar $\mathrm{Pb} / \mathrm{Eu}$ abundance ratios form a plateau in the metallicity range $-2.3<[\mathrm{Fe} / \mathrm{H}]<-1.4$, and that $\mathrm{Pb} / \mathrm{Eu}$ increases towards higher metallicity. The increase in $\mathrm{Pb} / \mathrm{Eu}$ at $[\mathrm{Fe} / \mathrm{H}]>-1.4$ suggests a growing contribution to the stellar lead abundance from the s-process opening in AGB stars. Roederer et al. (2010) concluded that s-process material was not widely dispersed until the Galactic metallicity grew considerably, as high as $[\mathrm{Fe} / \mathrm{H}]=$ -1.4. This also means that lead in stars of lower metallicity should be of pure r-process origin. We note that despite the large scatter in the $\mathrm{Pb} / \mathrm{Eu}$ abundance ratios of the Roederer et al. (2010) stars with $-2.3<[\mathrm{Fe} / \mathrm{H}]<-1.4$, the ratios are, on average, 0.85 dex larger than the corresponding value in the strongly r-process enhanced star CS 31082-001 (Plez et al. 2004). With $[\mathrm{Eu} / \mathrm{Fe}]=1.6($ Hill et al. 2002), this star experienced enrichment from, probably, a single r-process source. Following the suggestion of Christlieb et al. (2004), we hereafter refer to stars with $[\mathrm{Eu} / \mathrm{Fe}]>+1$ and $[\mathrm{Ba} / \mathrm{Eu}]<0$ as $r$-II stars. The heavyelement abundance ratios of each r-II star are expected to reflect the relative yields of the r-process. Why is there such a large discrepancy for $\mathrm{Pb} / \mathrm{Eu}$ between CS 31082-001 and the $-2.3<[\mathrm{Fe} / \mathrm{H}]<-1.4$ stars?

In the observed spectra of MP stars, lead is inferred from the lines of $\mathrm{Pb}$. Owing to a relatively low ionization energy of $7.43 \mathrm{eV}$, lead is mostly ionized in stellar atmospheres with effective temperature hotter than $T_{\text {eff }}=4000 \mathrm{~K}$, and the number density of neutral lead can easily deviate from the population in thermodynamic equilibrium (TE), because of deviations in the mean intensity of ionizing radiation from the Planck function. All previous stellar $\mathrm{Pb}$ analyses have been made under the assumption of local thermodynamic equilibrium (LTE), and the results obtained should be checked for any departures from LTE.

The detection of thorium permits, in principle, a nucleochronometric age estimate of a star by comparing the observed Th-to-stable neutron-capture element-abundance ratios with the corresponding initial values provided by the r-process event just prior to the time when the star was born (see, for example, Sneden et al. 1996; Cayrel et al. 2001; Frebel et al. 2007). Given that the half-life of ${ }^{232} \mathrm{Th}$ is long, stellar thorium has to be measured very precisely, with abundance errors of no more than $0.05 \mathrm{dex}$, to ensure that the uncertainty in the star's age is smaller than 2.3 Gyr.

This study aims to improve the calculations of the $\mathrm{Pb}$ I and Th II lines in stellar atmospheres using non-local thermodynamic equilibrium (non-LTE) line formation and to evaluate the systematic abundance errors caused by the simplified LTE lineformation treatment. We constructed, for the first time, model atoms for $\mathrm{Pb}$ I and Th II. The non-LTE method and the departures from LTE for $\mathrm{Pb}$ I are described in Sect. 2. Our theoretical results were used to determine the solar lead abundance (Sect. 3) and to revise the $\mathrm{Pb}$ abundances of the Roederer et al. (2010) stellar sample (Sect.4). Having added the r-II star HE 15230901 (Frebel et al. 2007), we found that the $\mathrm{Pb} / \mathrm{Eu}$ abundance ratios of the two r-II stars are well-reproduced by the waitingpoint r-process model as presented by Roederer et al. (2009), while an additional source of lead, most probably, the s-process in AGB stars, is needed to explain the $\mathrm{Pb}$ abundances of the stars with $[\mathrm{Fe} / \mathrm{H}]>-2.3$. The non-LTE calculations for Th II are presented in Sect. 5. Our conclusions are given in Sect. 6.

\section{Non-LTE calculations for $\mathrm{Pb}$ I}

To solve the coupled radiative transfer and statistical equilibrium (SE) equations, we used a revised version of the DETAIL program (Butler \& Giddings 1985) based on the accelerated lambda iteration (ALI) method described by Rybicki \& Hummer (1991, 1992). The update was presented by Mashonkina et al. (2011). The non-LTE calculations were performed with the MARCS model atmospheres (Gustafsson et al. 2008) ${ }^{2}$.

\subsection{Pb I atomic structure calculations}

The atomic structure of neutral lead has been well-studied with laboratory measurements. The ground level of $\mathrm{Pb}$ is $6 \mathrm{~s}^{2} 6 \mathrm{p}^{2}{ }^{3} \mathrm{P}_{0}$, and the ionization of the $6 \mathrm{p}$ electron gives the ionic core (Pb II) $6 \mathrm{~s}^{2} 6 \mathrm{p}$, which splits into two levels, ${ }^{2} \mathrm{P}_{1 / 2}^{\circ}$ (lower ionization limit $59819.57 \mathrm{~cm}^{-1}$ ) and ${ }^{2} \mathrm{P}_{3 / 2}^{\circ}$ (upper ionization limit $73900.64 \mathrm{~cm}^{-1}$ ) in the LS coupling scheme. The Pb I energy structure more closely approaches the $J J$ than the $L S$ coupling. Thus, the ground configuration $6 \mathrm{~s}^{2} 6 \mathrm{p}^{2}$ possesses five energy levels, which are designated as $(1 / 2,1 / 2)_{0},(1 / 2,3 / 2)_{1},(3 / 2,1 / 2)_{2}$, $(3 / 2,3 / 2)_{2}$, and $(3 / 2,3 / 2)_{0}$. The energy separation between the $6 s^{2} 6 p^{2}$ levels is remarkably large, for example, the second and the fifth levels have an excitation energy of $E_{\text {exc }}=0.97 \mathrm{eV}$ and $3.65 \mathrm{eV}$, respectively. Almost all the remaining known energy levels of $\mathrm{Pb}$ I belong to the $6 \mathrm{~s}^{2} 6 \mathrm{p} n$ l electronic configurations. Hereafter, $6 \mathrm{~s}^{2}$ is omitted in the configuration designations.

The early data on the $\mathrm{Pb}$ I energy levels obtained before 1958 were summarized by Moore (1958). After that, the analysis was greatly extended using classical emission (Wood \& Andrew 1968) and absorption (Brown et al. 1977) spectroscopy, as well as selective laser excitation (the papers relevant to our study are cited below). The measurements of long Rydberg series terminating on the first ionization limit provided many odd parity levels of the $6 \mathrm{p} n \mathrm{~s}$ and $6 \mathrm{p} n \mathrm{~d}$ (Brown et al. 1977, up to $n=59$ and $n=77$, respectively), and 6png ( $n=5-9$ in Wood \& Andrew 1968; and $n=21-47$ in Dembczyński et al. 1994) configurations. Even parity levels of the $6 \mathrm{p} n \mathrm{p}(n=7-60)$ and $6 \mathrm{p} n \mathrm{f}(n=5-56)$ configurations were measured by Hasegawa $\&$ Suzuki (1996) for a total of 205 energy levels with a total angular momentum $J=0,1$, and 2 . Information on several hundred autoionizing levels above the first ionization limit is now available (see Ahad et al. 2005, and references therein).

The data on the transition probabilities for $\mathrm{Pb}$ I available in the literature (Biémont et al. 2000; Fuhr \& Wiese 1992) are incomplete, and we rely on oscillator strengths computed in this study. The atomic structure of $\mathrm{Pb}_{\mathrm{I}}$ was calculated using the Cowan code (Cowan 1981). The 22 even parity interacting configurations $6 \mathrm{p}^{2}+6 \mathrm{p} n \mathrm{p}(7 \leq n \leq 15)+6 \mathrm{p} n \mathrm{f}(5 \leq n \leq 12)+$ $6 p 6 h+6 p 7 h+6 s 6 p^{2} 7 s+6 p^{4}$ were included in the energy matrix, while the odd set was restricted to 24 configurations, i.e., $6 \mathrm{p} n \mathrm{~s}(7 \leq n \leq 12)+6 \mathrm{p} n \mathrm{~d}(6 \leq n \leq 12)+6 \mathrm{p} n \mathrm{~g}(5 \leq n \leq$ 12) $+6 s 6 p^{2} 7 p+6 s 6 p^{3}+5 d^{9} 6 s^{2} 6 p^{3}$. The calculations generally follow the scheme used by Biémont et al. (2000). During the fitting of the calculated energy levels to the experimental ones, the number of varying energy parameters was restricted as much as possible. All the parameters describing the configurations in the $6 \mathrm{p} n \mathrm{l}$ series for different $n$ were varied collectively while the

2 http://marcs.astro.uu.se 
ratios of the corresponding ab initio values remained fixed. The exchange Slater integrals $\mathrm{G}^{k}$ within the configurations were also varied in a similar way. The electrostatic parameters including the configuration interaction parameters, which were not optimized in the fitting procedure, were scaled down by a factor of 0.80 , while the spin-orbit integrals were fixed at their ab initio values. As a result, the 67 even parity levels were described by the 25 parameters with a standard deviation of $73 \mathrm{~cm}^{-1}$ and the 51 odd parity levels by 21 parameters with a standard deviation of $74 \mathrm{~cm}^{-1}$. Our atomic structure calculations complemented the system of known energy levels in the region above $6.87 \mathrm{eV}$ with the 20 even parity levels of the $6 \mathrm{p} n \mathrm{f}(n=9-12, J=3$ and 4$)$ and $6 \mathrm{p} n \mathrm{~h}(n=6,7)$ configurations and 29 odd parity levels belonging mostly to the $6 \mathrm{p} n \mathrm{~g}(n=5-12)$ configurations.

The wavefunctions obtained after the fitting of energy levels were used to calculate the transition probabilities. The dipole transition integrals for all transitions were kept at ab initio Hartree-Fock values. The accuracy of our calculations was estimated by comparing the experimental data for common levels. Figure 1 shows that the difference in lifetime between our calculations and the measurements of Biémont et al. (2000) is mostly within $75 \%$, with the only outlier being the measured-tocalculated lifetime ratio of about 2.3. The predicted lifetimes are, on average, similar to the measured values. We note that the lifetimes of the highest 6pnd levels with $J=2$ depend critically on the position of the strongly interacting $6 s 6 \mathrm{p}^{35} \mathrm{~S}_{2}^{\circ}$ level. It seems that the only ${ }^{3} \mathrm{P}_{1}^{\circ}$ level of the $6 \mathrm{~s} 6 \mathrm{p}^{3}$ configuration is firmly located at $E_{\text {exc }}=85870 \mathrm{~cm}^{-1}$ (Krause et al. 1986; Müller et al. 1990). Dembczyński et al. (1994) estimated the position of the $6 \mathrm{~s} 6 \mathrm{p}^{3{ }^{3}} \mathrm{D}_{1}^{\circ}$ level at $E_{\text {exc }}=68943 \mathrm{~cm}^{-1}$ by analysing the interactions in the 6 pns and 6 pnd series. They also established that "the state $6 \mathrm{~s} 6 \mathrm{p}^{35} \mathrm{~S}_{2}^{\circ}$ does not occur as a dominant component in any of the electronic levels but is mixed into many odd levels". Its unperturbed value should be around $59300 \mathrm{~cm}^{-1}$. To predict the levels of the $6 \mathrm{~s} 6 \mathrm{p}^{3}$ configuration, the same scaling of ab initio values was applied to the parameters within the $6 \mathrm{p}^{3}$ subshell as for the $6 s^{2} 6 p^{2}$ configuration. The average energy and the $G^{1}(6 s, 6 p)$ parameter were adjusted to fit the energies of the ${ }^{3} \mathrm{P}_{1}^{\circ}$ and ${ }^{3} \mathrm{D}_{1}^{\circ}$ levels. The energy of the $6 \mathrm{~s} 6 \mathrm{p}^{3}{ }^{5} \mathrm{~S}_{2}^{\circ}$ level appeared to be $58700 \mathrm{~cm}^{-1}$, in quite good agreement with the estimates of Dembczyński et al. (1994). A similar consistency between theory and measurements was found for the even parity $6 \mathrm{p} n \mathrm{p}$ $(7 \leq n \leq 14)$ and 6 pnf $(6 \leq n \leq 8)$ levels when using the measured lifetimes of Li et al. (2001).

Figure 1 also shows the measured-to-calculated lifetime ratios from the calculations of Biémont et al. (2000), who took into account the core polarization effect that is expected to give rise to the precision of the predicted lifetimes. As can be seen, our results obtained without taking into account the core polarization agree very well with the calculations of Biémont et al. (2000). Therefore, $g f$-values calculated in this study are expected to have the same accuracy as the values in Biémont et al. (2000) for all the transitions in $\mathrm{Pb}$.

\subsection{Lead model atom}

Energy levels. To construct the model atom, we used all the measured and predicted levels in $\mathrm{Pb}$ I, which are shown in Fig. 2. The high-excitation $\left(E_{\mathrm{exc}} \geq 6.04 \mathrm{eV}\right)$ levels with common parity and energy separation of smaller than $0.01 \mathrm{eV}$ were combined into a single level. The final model atom is fairly complete. It includes 97 levels of $\mathrm{Pb}$ I and the ground state $6 \mathrm{~s}^{2} 6 \mathrm{p}^{2} \mathrm{P}_{1 / 2}^{\circ}$ of $\mathrm{Pb}$ II. The second sublevel of the ground term, ${ }^{2} \mathrm{P}_{3 / 2}^{\circ}\left(E_{\mathrm{exc}}=1.75 \mathrm{eV}\right)$, was

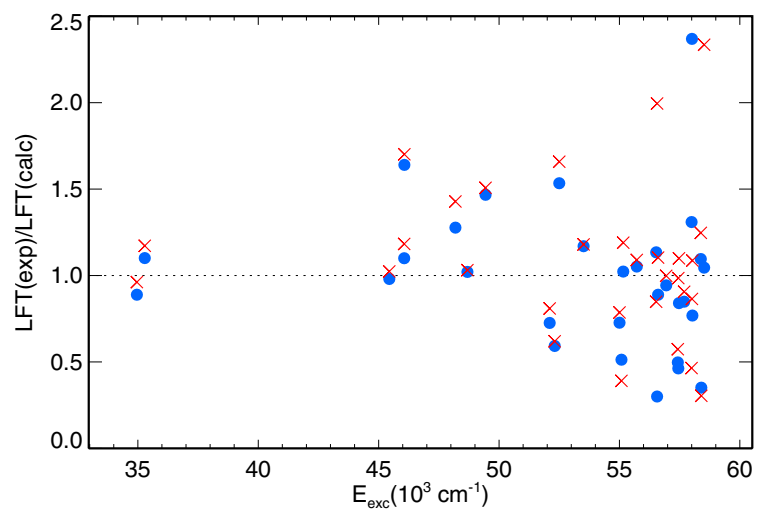

Fig. 1. Measured (Biémont et al. 2000) to calculated $\mathrm{Pb}$ I lifetime ratios for the $6 \mathrm{p} n \mathrm{~s}(7 \leq n \leq 12)$ and $6 \mathrm{p} n \mathrm{~d}(6 \leq n \leq 13)$ levels, mostly with $J=1$ and 2 . Open circles and crosses correspond to our and the Biémont et al. (2000) calculations, respectively.

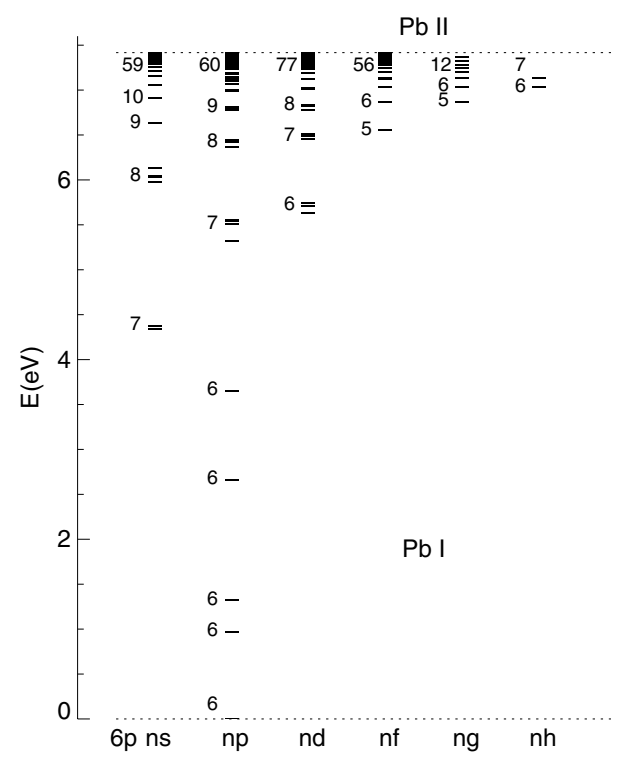

Fig. 2. Measured and predicted energy levels of $\mathrm{Pb}$ I that form our final $\mathrm{Pb}$ model atom.

taken into account only in the number conservation equation. The excited electronic configurations of $\mathrm{Pb}$ II produce levels with an excitation energy of more than $7.2 \mathrm{eV}$, and were thus ignored.

Radiative rates. For 141 transitions in $\mathrm{Pb}$ I connecting the $6 \mathrm{p}^{2}$ and $6 \mathrm{p} 7 \mathrm{p}$ levels to the $6 \mathrm{p} n \mathrm{~s}$ and $6 \mathrm{p} n \mathrm{~d}(n \leq 13)$ ones, we employed accurate $g f$-values based on measured natural radiative lifetimes and theoretical branching ratios from Biémont et al. (2000) and Fuhr \& Wiese (1992). For the remaining majority of transitions, we used oscillator strengths computed in this study. In total, 1954 radiative bound-bound $(b-b)$ transitions in $\mathrm{Pb}$ I were included in our SE calculations.

For the $\mathrm{Pb}$ I ground state, we applied the photoionization cross-sections $\sigma_{\mathrm{ph}}$ measured by Heppinstall \& Marr (1969) between the ionization threshold $(1671 \AA)$ and $1470 \AA$. For the remaining levels, we computed $\sigma_{\mathrm{ph}}$ using the hydrogen approximation formula with $n=n_{\mathrm{eff}}$, where $n_{\mathrm{eff}}=Z \sqrt{\chi_{\mathrm{H}} / \chi_{i}}$ is the effective principal quantum number for the level with the ionization energy $\chi_{i}$. Here, $Z=1$ and $\chi_{\mathrm{H}}$ is the hydrogen ionization energy. This choice was justified by the comparison of the experimental threshold cross-section $\sigma_{\text {threxp }}=10 \mathrm{Mb}$ for the $\mathrm{Pb}$ I ground state with the cross-sections calculated with various $n$. The use of $n=6$ results in the too low hydrogen approximation cross- 
section $\sigma_{\text {thr,hyd }}=0.0007 \mathrm{Mb}$. For $n_{\text {eff }}=1.35$, we computed $\sigma_{\text {thr,hyd }}=1.3 \mathrm{Mb}$, which is much closer to the experimental data, but a factor of ten lower.

Collisional rates. All levels in our model atom are coupled via collisional excitation and ionization by both electrons and neutral hydrogen atoms. The calculations of collisional rates rely on theoretical approximations. For electron-impact excitation, we used the formula of van Regemorter (1962) for the allowed transitions and assumed the effective collision strength $\Upsilon=1$ for the forbidden transitions. Electron-impact ionization crosssections were computed according to Drawin (1961).

For collisions with $\mathrm{H}_{\mathrm{I}}$ atoms, we employed the classical Drawin formula described by Steenbock \& Holweger (1984). Over the past few decades, this formula has been criticized for significantly overestimating the collision rates (see Barklem et al. 2011, and references therein). At the same time, the need for a thermalizing process not involving electrons in the atmospheres of, in particular, very metal-poor stars, has been indicated by many non-LTE spectral line formation studies (see Mashonkina et al. 2011, and references therein). Since no accurate calculations of either inelastic collisions of lead with neutral hydrogen atoms or other types of processes are available, we simulated an additional source of thermalization in the atmospheres of cool stars by using parametrized H I collisions. The Drawin formula can only be applied to allowed $b-b$ and $b-f$ transitions. For the forbidden transitions, a simple relation between hydrogen and electron collision rates, $C_{\mathrm{H}}=C_{\mathrm{e}} \sqrt{\left(m_{\mathrm{e}} / m_{\mathrm{H}}\right)} N_{\mathrm{H}} / N_{\mathrm{e}}$, was used following Takeda (1995). The SE calculations were performed with various efficiencies of collisions with $\mathrm{H}_{\mathrm{I}}$ atoms by applying a scaling factor of $S_{\mathrm{H}}=0$ (no hydrogen collisions), 0.1 , and 1.

We discuss below the effects of the uncertainty in the adopted photoionization cross-sections and collisional rates on our final results.

\subsection{Departures from LTE for $\mathrm{Pb}$ I}

We first inspect the statistical equilibrium of lead in the two model atmospheres representing the solar atmosphere, with $T_{\text {eff }} / \log g /[\mathrm{Fe} / \mathrm{H}]=5780 / 4.44 / 0$, and the atmosphere of a typical very metal-poor (VMP) cool giant (4500/1.0/-3) from the calculations with $S_{\mathrm{H}}=0.1$. The departure coefficients, $b_{i}=$ $n_{i}^{\mathrm{NLTE}} / n_{i}^{\mathrm{LTE}}$, for the $\mathrm{Pb}$ I and $\mathrm{Pb}$ II levels are shown in Fig. 3 , where $n_{i}^{\mathrm{NLTE}}$ and $n_{i}^{\mathrm{LTE}}$ are the statistical equilibrium and TE number densities, respectively. In these computations, the $\mathrm{Pb}$ abundance was adopted at $\log \varepsilon_{\mathrm{Pb}}=1.92$ in the solar model and at $\log \varepsilon_{\mathrm{Pb}}=-0.08([\mathrm{~Pb} / \mathrm{Fe}]=+1)$ in the VMP model. In each model, lead is almost completely ionized, such that the fraction of $\mathrm{Pb}$ I nowhere exceeds $4 \%$ in the solar atmosphere and $0.6 \%$ in the cool VMP atmosphere. This explains why the non-LTE mechanisms for $\mathrm{Pb}$ I are similar in the two models. We summarize our findings as follows.

1. In the atmosphere outside $\log \tau_{5000}=+0.2$ and +0.5 for the solar and VMP models, respectively, the most populated $6 \mathrm{p}^{2}$ levels of $\mathrm{Pb}$ I are underpopulated relative to their TE number densities and are close together. The main non-LTE mechanism is the ultraviolet (UV) overionization caused by superthermal radiation of a non-local origin below the thresholds at $\lambda_{\text {thr }}=1923,2033$, and $2606 \AA$ for the three excited $6 \mathrm{p}^{2}$ levels.

2. The populations of the $6 \mathrm{p} 7 \mathrm{~s}$ levels labelled in the model atom as $67 \mathrm{~s}$ and $77 \mathrm{~s}$ are controlled by the two mechanisms. The UV overionization $\left(\lambda_{\mathrm{thr}}=4022\right.$ and $\left.4076 \AA\right)$ tends to drain
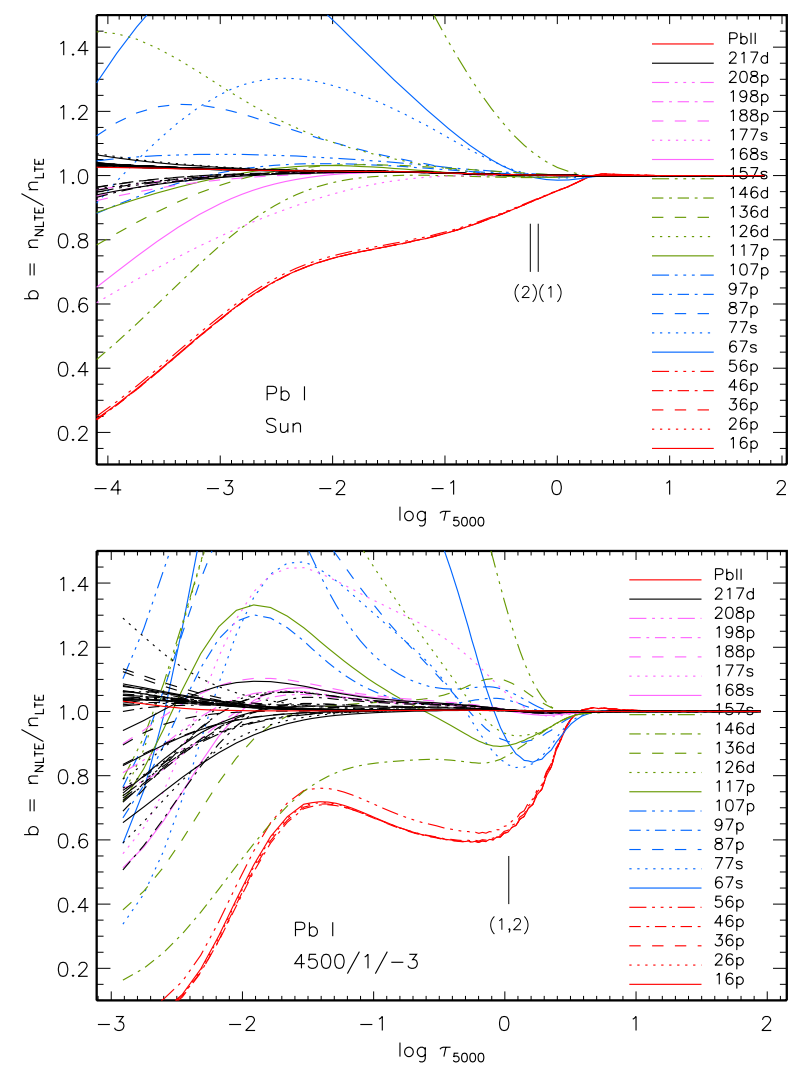

Fig. 3. Departure coefficients, $b$, for the lowest 60 levels of $\mathrm{Pb}$ I and the ground state of $\mathrm{Pb}$ II as a function of $\log \tau_{5000}$ in the model atmospheres 5780/4.44/0 (top panel) and 4500/1.0/-3 (bottom panel). The first 21 levels are quoted in the right part of each panel. For all the higher excitation levels, their behaviour is very similar to that of the $188 \mathrm{p}$ to $217 \mathrm{~d}$ levels, with $b \simeq 1$ in the solar and VMP models inside $\log \tau_{5000}=-3$ and -1 , respectively. $S_{\mathrm{H}}=0.1$ was used throughout. The vertical lines indicate the locations of the line core formation depths for $\mathrm{Pb}$ I 4057 (1) and $3683 \AA$ (2). See text for more details.

the populations far within the atmosphere. The competing process is radiative pumping of the strong $6 \mathrm{p}^{2}-6 \mathrm{p} 7 \mathrm{~s}$ transitions $(1 / 2,1 / 2)_{0}-(1 / 2,1 / 2)_{1}{ }^{\circ}(16 \mathrm{p}-77 \mathrm{~s}$ in our denotations, which produces the line at $2833 \AA),(1 / 2,3 / 2)_{1}-(1 / 2,1 / 2)_{0}{ }^{\circ}$ $(26 \mathrm{p}-67 \mathrm{~s}, 3683 \AA)$, and $(1 / 2,3 / 2)_{1}-(1 / 2,1 / 2)_{1}{ }^{\circ}(26 \mathrm{p}-77 \mathrm{~s}$, $3639 \AA$ ) by the ultraviolet $J_{v}-B_{v}\left(T_{\mathrm{e}}\right)$ excess radiation, which tends to produce enhanced excitation of the upper levels in the atmospheric layers where the line wing optical depth drops below 1 . The net effect is that the $67 \mathrm{~s}$ and $77 \mathrm{~s}$ levels are underpopulated far inside the atmosphere, at $\log \tau_{5000}=-0.7$ to +0.2 and -0.3 to +0.2 in the models 4500/1.0/-3 and 5780/4.44/0, respectively, and overpopulated in the higher layers. No process seems to compete with the radiative pumping of the $26 \mathrm{p}-157 \mathrm{~s}(2476 \AA)$ and $36 \mathrm{p}-157 \mathrm{~s}(2663 \AA)$ transitions resulting in a significant overpopulation of the upper level in the atmosphere outside $\log \tau_{5000}=+0.2$.

3. The overpopulation of the $6 \mathrm{p} 7 \mathrm{~s}$ levels is redistributed to the $6 \mathrm{p} 7 \mathrm{p}$ levels through inelastic collisions.

4. All the high-excitation levels with $E_{\text {exc }} \geq 6.1 \mathrm{eV}$ (starting from the 18th level in the model atom) couple thermally to the $\mathrm{Pb}$ II ground state.

5. $\mathrm{Pb}$ II represents the state in which the majority of the element exists and therefore the TE population is preserved. 
Table 1. Non-LTE abundance corrections (dex) for the $\mathrm{Pb}$ I $4057 \AA$ and Eu II $4129 \AA$ A lines from the calculations with $S_{\mathrm{H}}=0.1$.

\begin{tabular}{lcccc}
\hline \hline$T_{\text {eff }}$ & $\log g$ & {$[\mathrm{Fe} / \mathrm{H}]$} & \multicolumn{2}{c}{$\Delta_{\text {NLTE }}$} \\
\cline { 3 - 5 } & & & $\mathrm{Pb}$ I & \multicolumn{1}{c}{ Eu II } \\
\hline 5780 & 4.44 & 0 & 0.16 & 0.03 \\
\multicolumn{2}{c}{ [Pb/Fe] $=$} & $0.5^{a},[\mathrm{Eu} / \mathrm{Fe}]=0.7^{a}$ \\
4750 & 1.5 & -1 & 0.26 & 0.06 \\
5000 & 3.0 & -1 & 0.32 & 0.06 \\
5000 & 4.0 & -1 & 0.28 & 0.05 \\
5000 & 4.5 & -1 & 0.22 & 0.04 \\
5500 & 4.0 & -1 & 0.31 & 0.05 \\
5500 & 4.5 & -1 & 0.27 & 0.05 \\
4000 & 0.0 & -2 & 0.38 & 0.12 \\
4000 & 0.5 & -2 & 0.32 & 0.08 \\
4250 & 0.5 & -2 & 0.33 & 0.07 \\
4250 & 1.0 & -2 & 0.30 & 0.06 \\
4500 & 1.0 & -2 & 0.30 & 0.06 \\
4500 & 1.5 & -2 & 0.32 & 0.06 \\
4750 & 1.5 & -2 & 0.37 & 0.07 \\
4750 & 2.0 & -2 & 0.39 & 0.07 \\
5250 & 2.5 & -2 & 0.52 & 0.10 \\
{$[\mathrm{~Pb} / \mathrm{Fe}]=1.0^{a},[\mathrm{Eu} / \mathrm{Fe}]=1.8^{a}$} \\
4500 & 1.0 & -3 & 0.41 & 0.04 \\
4500 & 1.5 & -3 & 0.42 & 0.04 \\
4750 & 1.0 & -3 & 0.49 & 0.06 \\
4750 & 1.5 & -3 & 0.53 & 0.06 \\
5000 & 1.5 & -3 & 0.62 & 0.07 \\
\hline
\end{tabular}

Notes. ${ }^{(a)}$ Abundances used in non-LTE calculations.

The formation depth was specified as the depth where $\tau_{v} \lambda_{v}^{1 / 2}=$ 1 , following Mihalas (1978), where $\lambda_{v}$ is the ratio of the thermal to total absorption coefficient. Everywhere in the solar atmosphere, $\lambda_{v} \simeq 1$, irrespective of the frequency point. In the cool VMP model, $\lambda_{v}<1$ owing to significant contribution of Rayleigh scattering to the total opacity. For example, at the depth point $\log \tau_{5000}=0, \lambda_{v} \simeq 0.7$ at $4057 \AA$ and decreases towards shorter wavelengths. Even lower $\lambda_{v}$ values are obtained in the higher atmospheric layers.

The two lines, $\mathrm{Pb}_{\mathrm{I}} 4057 \AA \quad\left(6 \mathrm{p}^{2} \quad(1 / 2,3 / 2)_{2}-6 \mathrm{p} 7 \mathrm{~s}\right.$ $(1 / 2,1 / 2)_{1}{ }^{\circ}$ denoted as $36 \mathrm{p}-77 \mathrm{~s}$ in our model atom) and $3683 \AA$ $\left(6 \mathrm{p}^{2}(1 / 2,3 / 2)_{1}-6 \mathrm{p} 7 \mathrm{~s}(1 / 2,1 / 2)_{0}^{\circ}\right.$ denoted as $\left.26 \mathrm{p}-67 \mathrm{~s}\right)$, and, in many cases, only one of them are used in stellar $\mathrm{Pb}$ abundance analyses. In each model, non-LTE leads to a weakening of the $\mathrm{Pb}$ I lines relative to their LTE strengths and thus positive non-LTE abundance corrections. This is due to overionization $\left(b_{1}<1\right)$ and also the line source function $S_{\mathrm{lu}} \simeq b_{\mathrm{u}} / b_{1} B_{v}$ rises $\left(b_{\mathrm{u}}>b_{1}\right)$ being above the Planck function $B_{v}$ in the line-formation layers (Fig. 3), where $b_{\mathrm{u}}$ and $b_{1}$ are the departure coefficients of the upper and lower levels, respectively. We found that the departures from LTE are significantly larger in the VMP model than the solar one. The non-LTE abundance correction, $\Delta_{\mathrm{NLTE}}=\log \varepsilon_{\mathrm{NLTE}}-\log \varepsilon_{\mathrm{LTE}}$, decreases from +0.21 down to +0.09 dex for $\mathrm{Pb}_{\mathrm{I}} 3683 \AA$ in the solar model when $S_{\mathrm{H}}$ moves from 0 to 1 . Similar values, +0.19 and $+0.11 \mathrm{dex}$, were obtained for $\mathrm{Pb}$ I $4057 \AA$. In the $4500 / 1 /-3$ model, $\Delta_{\text {NLTE }}\left(\mathrm{Pb}_{\mathrm{I}} 4057 \AA\right)=+0.49$ and +0.38 dex when $S_{\mathrm{H}}=0$ and 1 , respectively.

Using $S_{\mathrm{H}}=0.1$, we computed $\Delta_{\mathrm{NLTE}}$ for the grid of model atmospheres that is characteristic of the Galactic halo stars. Table 1 presents the resulting values for $\mathrm{Pb}_{\text {I }} 4057 \AA$. It can be seen that the non-LTE correction increases toward lower metallicity from $\Delta_{\mathrm{NLTE}}=0.16 \mathrm{dex}$ in the solar model up to $\Delta_{\mathrm{NLTE}}=0.62 \mathrm{dex}$ in the $5000 / 1.50 /-3$ one. For a given metallicity, $\Delta_{\text {NLTE }}$ increases with increasing $T_{\text {eff }}$ and decreasing $\log g$.

In addition to different prescriptions of the collisions with hydrogen atoms, we performed two different test calculations for the $4500 / 1 /-3$ model to assess the influence of crucial atomic data on final results, where we used $S_{\mathrm{H}}=0.1$. First, we varied photoionization cross-sections by employing the principal quantum number $n$ instead of $n_{\mathrm{eff}}$ in the hydrogen approximation formula. For the $6 \mathrm{p}^{2}$ and $6 \mathrm{p} 7 \mathrm{~s}$ levels, this resulted in the reduction of $\sigma_{\mathrm{ph}}$ by a factor of from 150 to 70. However, the effect on $\Delta_{\text {NLTE }}$ for $\mathrm{Pb}$ I $4057 \AA$ was found to be minor, of less than 0.01 dex, because the departures from LTE for this line are controlled by the $b-b$ radiative transitions between the $6 \mathrm{p}^{2}$ and $6 \mathrm{p} 7 \mathrm{~s}$ levels.

Second, we checked the atomic model, where $\mathrm{H}_{\mathrm{I}}$ collisions were taken into account for the allowed transitions, but were neglected for the forbidden ones. As expected, this resulted in strengthening the departures from LTE. For Pb I $4057 \AA$, $\Delta_{\text {NLTE }}$ grew from 0.41 dex up to 0.53 dex. Thus, poor knowledge of collisions with $\mathrm{H}$ I atoms is the main source of the uncertainty in the calculated non-LTE abundance corrections for the $\mathrm{Pb}$ I lines.

\section{Solar lead abundance}

As a test and first application of the Pb I model atom, we determined the lead abundance for the Sun. We used solar central intensity observations taken from the Kitt Peak Solar Atlas of Brault et al. (1972). The only useful line of Pb r at $3683.46 \AA$ lies in a rather crowded spectral region, and the element abundance was derived by applying the line-profile fitting method with the sIU program (Reetz 1991). We employed the semi-empirical solar model atmosphere of Holweger \& Müller (1974, hereafter, HM74) and the theoretical MARCS model (Gustafsson et al. 2008 ) with $T_{\text {eff }}=5780 \mathrm{~K}$ and $\log g=4.44$. A depth-independent microturbulence of $0.9 \mathrm{~km} \mathrm{~s}^{-1}$ was adopted.

The continuum level in the spectral range 3678-3688 was fixed using an atomic line list from the VALD database (Kupka et al. 1999) and a molecular line list from Kurucz (1994). Our synthetic intensity profiles were convolved with a radial-tangential macroturbulence profile of $V_{\mathrm{mac}}=2.5 \mathrm{~km} \mathrm{~s}^{-1}$. Figure 4 illustrates the fit to the $3683 \AA$ blend, in particular, $\mathrm{Pb}_{\mathrm{I}} 3683.46 \AA$ and the strong Fe 3683.612 and $3683.629 \AA$ lines. For Pb I $3683.46 \AA$, $\log g f=-0.52$ (Biémont et al. 2000) was employed throughout.

Under the LTE assumption, we obtained $\log \varepsilon_{\mathrm{Pb}, \mathrm{LTE}}=$ 1.96 with the HM74 model and $\log \varepsilon_{\mathrm{Pb}, \mathrm{LTE}}=1.85$ with the solar MARCS model. It should be stressed that the solarphotosphere lead LTE abundance is lower than the meteoritic one $\log \varepsilon_{\mathrm{Pb}, \text { met }}=2.04 \pm 0.03$ (Asplund et al. 2009) and $2.06 \pm 0.03$ (Lodders et al. 2009), independent of the applied model atmosphere. The two most recent LTE determinations yielded $\log \varepsilon_{\mathrm{Pb}, \mathrm{LTE}}=1.75 \pm 0.10$ based on the theoretical threedimensional (3D) model (Asplund et al. 2009) and $2.00 \pm 0.06$ based on the HM74 model (Lodders et al. 2009).

We found that non-LTE decreases the discrepancy between the solar photosphere and meteoritic $\mathrm{Pb}$ abundance. When using the HM74 model, the non-LTE abundances amount to $\log \varepsilon_{\mathrm{Pb}}=$ $2.12,2.09$, and 2.03 from the calculations with $S_{\mathrm{H}}=0,0.1$, and 1 , respectively. Figure 4 shows the best non-LTE fit for $S_{\mathrm{H}}=0.1$. For the solar MARCS model, the non-LTE abundances are 2.00, 1.97, and 1.92 depending on the used $S_{\mathrm{H}}$ value, i.e., for $0,0.1$, and 1 , respectively. We note that, in all cases, the non-LTE correction calculated for the emergent intensity spectrum is smaller 


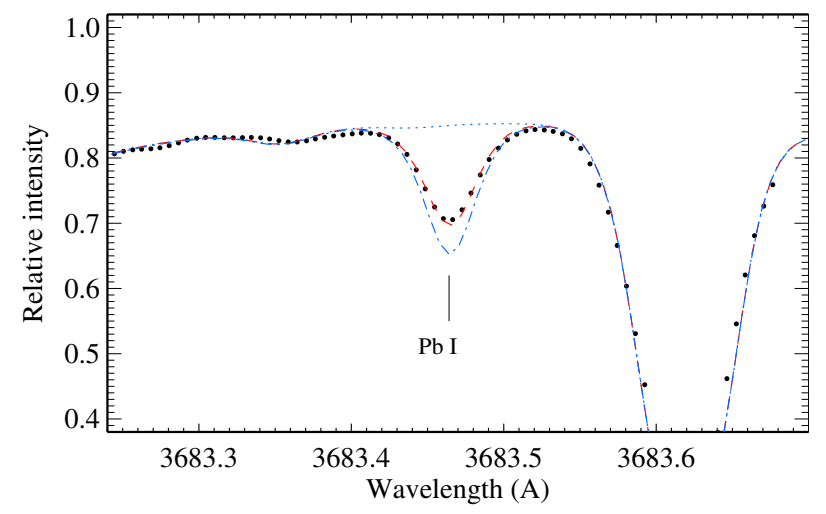

Fig. 4. Synthetic non-LTE $\left(S_{\mathrm{H}}=0.1\right.$, dashed curve) and LTE (dashdotted curve) disk-center intensity profiles of $\mathrm{Pb}_{\mathrm{I}} 3683.46 \AA$ computed with the HM74 model atmosphere and $\log \varepsilon_{\mathrm{Pb}}=2.09$ compared with the observed spectrum of the Brault et al. (1972) solar atlas (bold dots). Dotted curve corresponds to the atmosphere without lead.

than that for the flux spectrum. This is because the disk-center radiation emerges from deeper layers, where the departures from LTE are weaker. From the analysis of the Pb r $3683 \AA$ line alone, we cannot determine the efficiency of poorly known inelastic collisions with $\mathrm{H}_{\mathrm{I}}$ atoms in SE calculations, and we choose $S_{\mathrm{H}}=$ 0.1 based on our empirical estimates for $\mathrm{Ca}$ I-Ca II and $\mathrm{Fe}$ I-Fe II (Mashonkina et al. 2007, 2011). Thus, our final solar lead abundance is $\log \varepsilon_{\mathrm{Pb}, \mathrm{NLTE}}=2.09$ with the HM74 model atmosphere and $\log \varepsilon_{\mathrm{Pb}, \mathrm{NLTE}}=1.97$ with the solar MARCS model.

\section{Constraints on the $\mathrm{Pb}$ production mechanisms in the early Galaxy}

We revise the $\mathrm{Pb}$ abundances of metal-poor stars available in the literature and investigate the metallicity behaviour of the $\mathrm{Pb} / \mathrm{Eu}$ abundance ratio. This ratio is particularly sensitive to whether the production of lead occurred in a pure r-process or there was a non-negligible s-process contribution. The r-nuclei of lead are produced through several decay channels. At the time when the r-process event stops (likely after a few seconds), there is the direct $\beta^{-}$decay of very neutron-rich isobaric nuclei with $A=$ ${ }^{206-208}$ to ${ }^{206} \mathrm{~Pb},{ }^{207} \mathrm{~Pb}$, and ${ }^{208} \mathrm{~Pb}$. Then there is the $\alpha$ - and $\beta$-decay of nuclei with $210 \leq A \leq 231$ and $A=234$ back to $\mathrm{Pb}$, which operates shortly after the termination of the r-process, within few million years. And finally the radioactive decay of the long-lived $\mathrm{Th}$ and $\mathrm{U}$ isotopes produced $\mathrm{Pb}$ over the course of almost the entire age of the Galaxy. As stressed by Roederer et al. (2009), no $\mathrm{Pb}$ isotopes can be measured individually owing to the relatively small isotope shifts $(\leq 0.02-0.03 \AA)$ and overall weakness of the $\mathrm{Pb}$ I lines.

Hence, for the total elemental abundances, the classical waiting-point (WP) r-process model of Kratz et al. (2007) predicts that $\log \varepsilon_{\mathrm{r}, 0}(\mathrm{~Pb} / \mathrm{Eu})=0.61$ in a newly born star and $\log \varepsilon_{\mathrm{r}, 13}(\mathrm{~Pb} / \mathrm{Eu})=0.70$ in a $13 \mathrm{Gyr}$ old star (Roederer et al. 2009). Another estimate of a pure r-process production ratio can be obtained using the solar r-residuals. With an s-process contribution to the solar $\mathrm{Pb}$ of $81 \%$ (Travaglio et al. 2001) ${ }^{3}$ and to the solar Eu of $6 \%$ (Travaglio et al. 1999), we computed the Solar System r-process ratio $\log \varepsilon_{\mathrm{SSr}}(\mathrm{Pb} / \mathrm{Eu})=0.82$. When

\footnotetext{
3 These authors in reality indicated $91 \%$. However, using s-fractions of $90,60,77$, and $89 \%$ for the ${ }^{204} \mathrm{~Pb},{ }^{206} \mathrm{~Pb},{ }^{207} \mathrm{~Pb}$, and ${ }^{208} \mathrm{~Pb}$ isotopes, respectively, from their Table 3, we calculated an s-fraction of $81 \%$ for the total lead abundance.
}

computing s-fractions, Travaglio et al. $(1999,2001)$ used the meteoritic isotope abundances from Anders \& Grevesse (1989). For comparison, the ratio of the meteoritic total abundances is $\log \varepsilon_{\text {met }}(\mathrm{Pb} / \mathrm{Eu})=1.51$ and 1.53 according to Anders \& Grevesse (1989) and Lodders et al. (2009), respectively.

The largest data set on stellar $\mathrm{Pb}$ abundances was compiled by Roederer et al. (2010). Their data are based on the analysis of $\mathrm{Pb}_{\text {I }} 4057 \AA$. Before correcting these abundances, we excluded all stars with only upper limits to the $\mathrm{Pb}$ abundance, the carbonenhanced stars with $[\mathrm{C} / \mathrm{Fe}]>0.3$, and also VMP stars enriched in s-process material, with $\log \varepsilon(\mathrm{Pb} / \mathrm{Eu}) \geq+1.8$ at $[\mathrm{Fe} / \mathrm{H}]<-2$ (see small blue " $X$ " in their Fig. 3) from the Roederer et al. (2010) sample. When determining the carbon abundances of the stars to be checked, we also relied on the Simmerer et al. (2004) study, which was used by Roederer et al. (2010) to compile most of their stellar sample. In total, 49 stars were selected in the $-2.26 \leq[\mathrm{Fe} / \mathrm{H}]<-0.59$ metallicity range. We added to them the two r-II stars, i.e., CS 31082-001 (Hill et al. 2002; Plez et al. 2004) and HE 1523-0901 (Frebel et al. 2007, 2012, in prep.), and also the halo star HD 29907 from Sitnova \& Mashonkina (2011). The stars used are listed in Table 2 with stellar parameters and LTE element abundances taken from the cited papers and with non-LTE abundance corrections obtained in this study. The non-LTE calculations were performed with the MARCS model atmospheres (Gustafsson et al. 2008). Where necessary, the MARCS model structures were interpolated for given $T_{\text {eff }}$, $\log g$, and $[\mathrm{Fe} / \mathrm{H}]$ using a FORTRAN-based routine written by Thomas Masseron ${ }^{4}$. For each star, the SE calculations were performed using its given LTE element abundance.

For self-consistency, we also revised the europium abundances of the stars in our sample. The non-LTE calculations for Eu II were performed using the method treated by Mashonkina \& Gehren (2000). Everywhere, $S_{\mathrm{H}}=0.1$ was adopted. In the stellar parameter range with which we are concerned, non-LTE leads to weakened Eu II lines and positive abundance corrections. We computed $\Delta_{\text {NLTE }}$ for the seven lines, i.e., Eu II $3724,3819,4129$, and $4205 \AA$ with $E_{\text {exc }}=0$ and Eu II 3907,4435 , and $4522 \AA$ with $E_{\text {exc }}=0.21 \mathrm{eV}$. Table 1 presents the resulting values for Eu II $4129 \AA$ in the grid of model atmospheres characteristic of the Galactic halo stars. Table 2 does the same for the stars in our sample. For each line, $\Delta_{\text {NLTE }}$ increases toward lower metallicity and lower surface gravity, although, it is overall small and does not exceed 0.17 dex for different lines. For example, $\Delta_{\mathrm{NLTE}}$ ranges between 0.02 dex and 0.06 dex for different lines in the $5600 / 3.75 /-0.59$ model, between 0.04 dex and 0.14 dex in the 4825/1.5/-2.9 model, and between 0.05 dex and $0.17 \mathrm{dex}$ in the $4050 / 0.0 /-2.08$ model. For each model atmosphere, $\Delta_{\text {NLTE }}$ of Eu II $4129 \AA$ turned out to represent the mean non-LTE correction for all the computed lines. For a given star, we used it as the mean non-LTE correction and applied it to the observed europium LTE abundance.

The non-LTE and LTE $\mathrm{Pb} / \mathrm{Eu}$ abundance ratios are plotted in Fig. 5. As shown by Hill et al. (2002) for CS 31082-001 and Frebel et al. (2007) for HE 1523-0901, the heavy elements beyond the iron group in these stars originate from the r-process, and the observed element abundance ratios can be used to test theoretical nucleosynthesis models. We found that both stars have very similar $\mathrm{Pb} / \mathrm{Eu}$ abundance ratios at $\log \varepsilon_{\mathrm{NLTE}}(\mathrm{Pb} / \mathrm{Eu})=$ 0.67 and 0.68 , respectively, and that the WP r-process model for a 13 Gyr old star (Kratz et al. 2007; Roederer et al. 2009) perfectly reproduces the observations. We also stress that the $\mathrm{Pb} / \mathrm{Eu}$ abundance ratios of the r-II stars match the solar r-process

\footnotetext{
${ }^{4}$ http://marcs.astro.uu.se/software.php
} 
Table 2. Non-LTE and LTE abundances of $\mathrm{Pb}$ and Eu and non-LTE abundance corrections (dex) for the Pb I $4057 \AA$ and Eu II $4129 \AA$ lines in the Roederer et al. (2010) stellar sample.

\begin{tabular}{|c|c|c|c|c|c|c|c|c|c|}
\hline \multirow[b]{2}{*}{ Star } & \multirow[b]{2}{*}{$T_{\text {eff }}$} & \multirow[b]{2}{*}{$\log g$} & \multirow[b]{2}{*}[\mathrm{Fe}/\mathrm{H}]{} & \multicolumn{3}{|c|}{$\mathrm{Pb}$} & \multicolumn{3}{|c|}{$\mathrm{Eu}$} \\
\hline & & & & $\log \varepsilon_{\mathrm{NLTE}}{ }^{a}$ & $\log \varepsilon_{\mathrm{LTE}}$ & $\Delta_{\mathrm{NLTE}^{a}}{ }^{a}$ & $\log \varepsilon_{\mathrm{NLTE}}{ }^{a}$ & $\log \varepsilon_{\mathrm{LTE}}$ & $\Delta_{\mathrm{NLTE}}{ }^{a}$ \\
\hline BD+01 2916 & 4200 & 0.40 & -1.92 & 0.12 & -0.20 & 0.32 & -1.14 & -1.22 & 0.08 \\
\hline $\mathrm{BD}+191185$ & 5500 & 4.19 & -1.09 & 1.06 & 0.77 & 0.29 & -0.18 & -0.23 & 0.05 \\
\hline BD+29 2356 & 4760 & 1.60 & -1.59 & 0.65 & 0.35 & 0.30 & -0.62 & -0.69 & 0.07 \\
\hline BD+30 2611 & 4330 & 0.60 & -1.50 & 0.92 & 0.56 & 0.36 & -0.29 & -0.36 & 0.07 \\
\hline CS31082-001 & 4825 & 1.50 & -2.90 & 0.01 & -0.55 & 0.56 & -0.66 & -0.72 & 0.06 \\
\hline G009-036 & 5625 & 4.57 & -1.17 & 1.53 & 1.25 & 0.28 & -0.11 & -0.16 & 0.05 \\
\hline G028-043 & 5060 & 4.50 & -1.64 & 0.97 & 0.69 & 0.28 & -0.48 & -0.53 & 0.05 \\
\hline G029-025 & 5225 & 4.28 & -1.09 & 1.06 & 0.80 & 0.26 & -0.18 & -0.23 & 0.05 \\
\hline G058-025 & 6000 & 4.21 & -1.40 & 1.67 & 1.29 & 0.38 & -0.61 & -0.66 & 0.05 \\
\hline G059-001 & 5920 & 3.98 & -0.95 & 1.98 & 1.64 & 0.34 & -0.24 & -0.29 & 0.05 \\
\hline G068-003 & 4975 & 3.50 & -0.76 & 1.49 & 1.19 & 0.30 & 0.22 & 0.16 & 0.06 \\
\hline G095-057A & 4955 & 4.40 & -1.22 & 1.35 & 1.14 & 0.21 & -0.19 & -0.23 & 0.04 \\
\hline G102-020 & 5250 & 4.44 & -1.25 & 1.04 & 0.79 & 0.25 & -0.27 & -0.32 & 0.05 \\
\hline G102-027 & 5600 & 3.75 & -0.59 & 1.87 & 1.64 & 0.23 & 0.43 & 0.40 & 0.03 \\
\hline G113-022 & 5525 & 4.25 & -1.18 & 1.49 & 1.19 & 0.30 & -0.08 & -0.13 & 0.05 \\
\hline G122-051 & 4864 & 4.51 & -1.43 & 0.54 & 0.34 & 0.20 & -0.23 & -0.27 & 0.04 \\
\hline G123-009 & 5490 & 4.75 & -1.25 & 1.40 & 1.13 & 0.27 & -0.16 & -0.21 & 0.05 \\
\hline G126-036 & 5490 & 4.50 & -1.06 & 1.94 & 1.67 & 0.27 & 0.09 & 0.04 & 0.05 \\
\hline G140-046 & 4980 & 4.42 & -1.30 & 1.41 & 1.19 & 0.22 & -0.37 & -0.41 & 0.04 \\
\hline G179-022 & 5080 & 3.20 & -1.35 & 0.72 & 0.39 & 0.33 & -0.17 & -0.22 & 0.05 \\
\hline G221-007 & 5020 & 3.37 & -0.98 & 1.99 & 1.69 & 0.30 & -0.06 & -0.11 & 0.05 \\
\hline HD 3008 & 4250 & 0.25 & -2.08 & -0.21 & -0.56 & 0.35 & -1.02 & -1.09 & 0.07 \\
\hline HD 6755 & 5100 & 2.93 & -1.68 & 0.73 & 0.32 & 0.41 & -0.44 & -0.50 & 0.06 \\
\hline HD 6833 & 4400 & 1.50 & -0.85 & 1.93 & 1.69 & 0.24 & 0.15 & 0.10 & 0.05 \\
\hline HD 8724 & 4535 & 1.40 & -1.91 & 0.32 & 0.00 & 0.32 & -0.80 & -0.86 & 0.06 \\
\hline HD 23798 & 4450 & 1.06 & -2.26 & 0.09 & -0.21 & 0.30 & -1.30 & -1.36 & 0.06 \\
\hline HD 26297 & 4320 & 1.11 & -1.98 & 0.22 & -0.11 & 0.33 & -1.16 & -1.22 & 0.06 \\
\hline HD 29574 & 4250 & 0.80 & -2.00 & 0.25 & -0.06 & 0.31 & -0.57 & -0.63 & 0.06 \\
\hline HD $29907^{b}$ & 5500 & 4.64 & -1.55 & 1.03 & 0.70 & 0.33 & -0.45 & -0.50 & 0.05 \\
\hline HD 37828 & 4350 & 1.50 & -1.62 & 1.14 & 0.77 & 0.37 & -0.48 & -0.53 & 0.05 \\
\hline HD 44007 & 4850 & 2.00 & -1.72 & 0.67 & 0.31 & 0.36 & -0.87 & -0.94 & 0.07 \\
\hline HD 63791 & 4675 & 2.00 & -1.90 & 0.58 & 0.22 & 0.36 & -0.85 & -0.92 & 0.07 \\
\hline HD 74462 & 4700 & 2.00 & -1.52 & 0.79 & 0.49 & 0.30 & -0.32 & -0.39 & 0.07 \\
\hline HD 105755 & 5700 & 3.82 & -0.83 & 1.71 & 1.43 & 0.28 & 0.07 & 0.02 & 0.05 \\
\hline HD 106516 & 6170 & 4.21 & -0.81 & 1.85 & 1.56 & 0.29 & 0.05 & -0.04 & 0.09 \\
\hline HD 108317 & 5234 & 2.68 & -2.18 & 0.69 & 0.17 & 0.52 & -1.22 & -1.32 & 0.10 \\
\hline HD 121135 & 4934 & 1.91 & -1.54 & 0.72 & 0.38 & 0.34 & -0.63 & -0.70 & 0.07 \\
\hline HD 122956 & 4508 & 1.55 & -1.95 & 0.19 & -0.13 & 0.32 & -0.73 & -0.79 & 0.06 \\
\hline HD 141531 & 4360 & 1.14 & -1.79 & 0.52 & 0.20 & 0.32 & -0.66 & -0.72 & 0.06 \\
\hline HD 166161 & 5350 & 2.56 & -1.23 & 1.19 & 0.84 & 0.35 & -0.41 & -0.48 & 0.07 \\
\hline HD 171496 & 4950 & 2.37 & -0.67 & 1.60 & 1.41 & 0.19 & 0.17 & 0.11 & 0.06 \\
\hline HD 175305 & 4770 & 1.80 & -1.73 & 0.06 & -0.28 & 0.34 & -0.82 & -0.89 & 0.07 \\
\hline HD 187111 & 4270 & 1.05 & -1.97 & 0.21 & -0.10 & 0.31 & -0.82 & -0.88 & 0.06 \\
\hline HD 201891 & 5910 & 4.19 & -1.09 & 1.58 & 1.25 & 0.33 & -0.17 & -0.22 & 0.05 \\
\hline HD 204543 & 4670 & 1.49 & -1.87 & 0.41 & 0.05 & 0.36 & -0.98 & -1.05 & 0.07 \\
\hline HD 206739 & 4650 & 1.78 & -1.72 & 0.69 & 0.38 & 0.31 & -0.55 & -0.62 & 0.07 \\
\hline HD 210295 & 4750 & 2.50 & -1.46 & 1.04 & 0.72 & 0.32 & -0.27 & -0.34 & 0.07 \\
\hline HD 214925 & 4050 & 0.00 & -2.08 & -0.13 & -0.50 & 0.37 & -0.97 & -1.09 & 0.12 \\
\hline HD 220838 & 4300 & 0.60 & -1.80 & 0.39 & 0.05 & 0.34 & -0.86 & -0.93 & 0.07 \\
\hline HD 221170 & 4510 & 1.00 & -2.16 & 0.21 & -0.09 & 0.30 & -0.80 & -0.86 & 0.06 \\
\hline HD 235766 & 4650 & 1.20 & -1.93 & 0.42 & 0.10 & 0.32 & -0.80 & -0.86 & 0.06 \\
\hline HE $1523-0901^{c}$ & 4630 & 1.00 & -2.95 & 0.11 & $-0.34^{d}$ & 0.45 & -0.57 & -0.62 & 0.05 \\
\hline
\end{tabular}

Notes. ${ }^{(a)}$ From the calculations with $S_{\mathrm{H}}=0.1,{ }^{(b)}$ stellar parameters and LTE abundances from Sitnova \& Mashonkina (2011), ${ }^{(c)}$ stellar parameters and europium LTE abundance from Frebel et al. (2007), (d) Frebel et al. (in prep.).

ratio $\log \varepsilon_{\mathrm{SSr}}(\mathrm{Pb} / \mathrm{Eu})=0.82$ within the error bars. According to Plez et al. (2004), the uncertainty in the $\mathrm{Pb}$ abundance of CS 31082-001 is 0.15 dex. This provides strong evidence of universal $\mathrm{Eu}$ and $\mathrm{Pb}$ relative r-process yields during the Galactic history.
Despite the large scatter in the abundances, a clear upward trend is seen in the $\log \varepsilon(\mathrm{Pb} / \mathrm{Eu})-[\mathrm{Fe} / \mathrm{H}]$ plane for all stars with $[\mathrm{Fe} / \mathrm{H}]>-2.3$. The $\mathrm{Pb} / \mathrm{Eu}$ abundance ratio grows from $\log \varepsilon_{\text {NLTE }}(\mathrm{Pb} / \mathrm{Eu}) \simeq 0.8$ at $[\mathrm{Fe} / \mathrm{H}]=-2$ up to the solar value at $[\mathrm{Fe} / \mathrm{H}]=-0.6$. Following Roederer et al. (2010), we separated 
A\&A 540, A98 (2012)

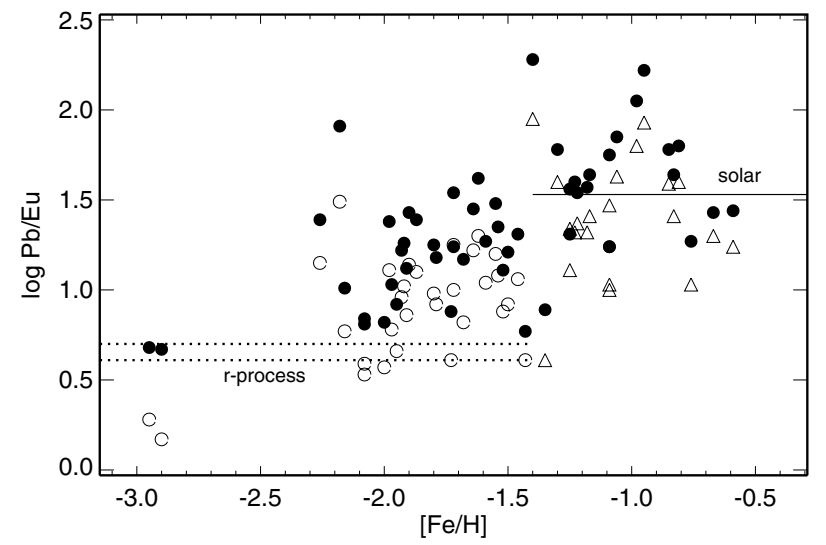

Fig. 5. Stellar non-LTE (filled circles) and LTE (open circles for the stars with $\log g \leq 3$ and triangles for $\log g>3$ ) $\mathrm{Pb} / \mathrm{Eu}$ abundance ratios as a function of metallicity. The solid line indicates the Solar system meteoritic value. The two dotted lines correspond to a pure r-process production of $\mathrm{Pb}$ and Eu predicted by the waiting-point $\mathrm{r}$-process model for a newly born (the low-lying curve) and 13 Gyr old (the up-lying curve) star as given by Roederer et al. (2009). The non-LTE abundances were calculated with $S_{\mathrm{H}}=0.1$.

these stars in two groups depending on their metallicity. The low-metallicity sample (hereafter, LMS) includes 29 stars in the $-2.26 \leq[\mathrm{Fe} / \mathrm{H}]<-1.40$ metallicity range, and the mildly metalpoor sample $(-1.4 \leq[\mathrm{Fe} / \mathrm{H}] \leq-0.59$, hereafter, MMPS $)$ includes 21 stars. It is worth noting that each subsample is highly biased in terms of surface gravity owing to a common selection effect, namely most LMS stars are cool giants, while the MMPS stars are mainly dwarf stars.

As seen in Fig. 5, the LMS stars have significantly larger $\mathrm{Pb} / \mathrm{Eu}$ ratios than the r-II stars, independent of either LTE or non-LTE. Excluding a clear outlier, i.e., the star HD 108317 $\left([\mathrm{Fe} / \mathrm{H}]=-2.18, \log \varepsilon_{\mathrm{NLTE}}(\mathrm{Pb} / \mathrm{Eu})=1.91\right)$, we calculated the mean $\log \varepsilon_{\text {LTE }}(\mathrm{Pb} / \mathrm{Eu})=0.93 \pm 0.23$ and $\log \varepsilon_{\mathrm{NLTE}}(\mathrm{Pb} / \mathrm{Eu})=$ $1.19 \pm 0.24$ for the $28 \mathrm{LMS}$ stars. We conclude that the s-process in AGB stars started to produce lead before the Galactic metallicity had reached $[\mathrm{Fe} / \mathrm{H}]=-2.3$. The low-metallicity s-process nuclesynthesis calculations (Roederer et al. 2010; Lugaro et al. 2012) do not contradict this observational finding. Roederer et al. (2010) presented the surface composition at the end of the AGB phase for a number of intermediate-mass models with $[\mathrm{Fe} / \mathrm{H}]=$ -2.3 and $M=4.5-6 M_{\odot}$. The minimum value produced by those models is $\log \varepsilon(\mathrm{Pb} / \mathrm{Eu})=1.9$. Given sufficient time, their contributions could result in an increase of the Galactic $\mathrm{Pb} / \mathrm{Eu}$ abundance ratio above the pure r-process value. Earlier, from analyses of the stellar (Ba, La, Nd)/(Eu, Dy) abundance ratios, Burris et al. (2000) and Simmerer et al. (2004) obtained evidence that the s-process might be active as early as $[\mathrm{Fe} / \mathrm{H}]=-2.3$ and -2.6 , respectively.

For the MMPS stars, the derived mean $\log \varepsilon_{\mathrm{NLTE}}(\mathrm{Pb} / \mathrm{Eu})=$ $1.58 \pm 0.31$ is very close to the Solar System value of $\log \varepsilon_{\text {met }}(\mathrm{Pb} / \mathrm{Eu})=1.53$ (Lodders et al. 2009). This supports the theoretical result of Travaglio et al. (2001), who predicted that the AGB stars with $[\mathrm{Fe} / \mathrm{H}] \simeq-1$ made the greatest contribution to the solar abundance of s-nuclei of lead. We note that LTE leads to a $\mathrm{Pb} / \mathrm{Eu}$ abundance ratio that is 0.22 dex lower for the MMPS stars. The star G 58-25, with $[\mathrm{Fe} / \mathrm{H}]=-1.40$ and $\log \varepsilon_{\mathrm{NLTE}}(\mathrm{Pb} / \mathrm{Eu})=2.28$, was not included in the mean calculations.

In general, our findings are independent of any variation in the stellar parameters that correspond to reasonable observational uncertainties and a variation in the $S_{\mathrm{H}}$ value. For example, for CS 31082-001, a change of $+100 \mathrm{~K}$ in $T_{\text {eff }}$ (a $\sim 2 \sigma$ error in Hill et al. 2002) produces a change in the calculated $\mathrm{Pb}$ non-LTE abundance of +0.16 dex. The Pb I $4057 \AA$ line is insensitive to a variation in the values of $\log \mathrm{g}$ and microturbulence velocity, $V_{\text {mic }}$. The total effect of varying $T_{\text {eff }}, \log g$ (by $+0.3 \mathrm{dex}$ ), and $V_{\text {mic }}$ (by $+0.2 \mathrm{~km} \mathrm{~s}^{-1}$ ) on the derived Eu abundance was estimated by Hill et al. (2002) as to be +0.12 dex. Thus, the $\mathrm{Pb} / \mathrm{Eu}$ abundance ratio is only slightly affected by the uncertainties in the stellar parameters. The non-LTE calculations performed for CS 31082-001 with $S_{\mathrm{H}}=0$ and 1 result in values of $\log \varepsilon(\mathrm{Pb} / \mathrm{Eu})$ that are only 0.04 and 0.02 dex lower than values for $S_{\mathrm{H}}=0.1$. To evaluate the uncertainty in the $\mathrm{Pb} / \mathrm{Eu}$ abundance ratios of the LMS and MMPS stars, we chose the two representative stars HD 3008 (4250/0.25/-2.08) and G 102020 (5250/4.44/-1.25). Simmerer et al. (2004) estimated that the stellar parameters of their sample are internally consistent to $\Delta T_{\text {eff }}=100 \mathrm{~K}, \Delta \log \mathrm{g}=0.25$, and $\Delta V_{\text {mic }}=0.1 \mathrm{~km} \mathrm{~s}^{-1}$. An upward revision of the stellar parameters for HD 3008 results in a cumulative change of $+0.11 \mathrm{dex}$ in $\log \varepsilon_{\mathrm{Pb}}$ and $+0.10 \mathrm{dex}$ in $\log \varepsilon_{\mathrm{Eu}}$. For G 102-020, the abundance errors caused by the uncertainties in the stellar parameters also have a common sign for $\mathrm{Pb}$ and Eu and amount to 0.09 and 0.06 dex, respectively.

We note that we did not consider the effects on the abundances of the use of 3D hydrodynamical model atmospheres. However, those effects were estimated for $\mathrm{Pb}$ I $4057 \AA$ ( $E_{\text {exc }}=$ $1.32 \mathrm{eV}$ ) and the Eu II lines arising from the ground state using the 3D-1D LTE abundance corrections calculated by Collet et al. (2007) and Hayek et al. (2011) for a sample of "fictitious" atomic (Na I, Mg I, $\mathrm{Ca}_{\mathrm{I}}, \mathrm{Fe}$ I, and $\mathrm{Fe}_{\text {II }}$ ) lines at selected wavelengths, with varying lower-level excitation potentials and line strengths. Overall, the difference between the abundances based on 3D and one-dimensional (1D) techniques (the 3D-1D correction) is negative for the lines of neutral atoms and decreases in absolute value with increasing $E_{\text {exc }}$. In the $4717 / 2.2 /-1$ models, they have similar values for all the chemical species, i.e., the $3 \mathrm{D}-1 \mathrm{D}$ correction varies between $-0.20 \mathrm{dex}$ and -0.28 dex for the lines with $E_{\text {exc }}=0$ and between -0.10 dex and -0.18 dex for $E_{\text {exc }}=2 \mathrm{eV}$. The $3 \mathrm{D}$ effects increase toward lower metallicity, however, with different rate for different atoms. In the $4732 / 2.2 /-2$ and 4858/2.2/-3 models, the 3D-1D corrections are similar for $\mathrm{Mg}$ I and $\mathrm{Fe}$ I, but different from that for $\mathrm{Na}$ I and $\mathrm{Ca}$ I. For the weak Na I line with $E_{\mathrm{exc}}=1.3 \mathrm{eV}$ and an equivalent width of $E W<20 \mathrm{~m} \AA, 3 \mathrm{D}-1 \mathrm{D} \simeq-0.12$ dex in the $-3 \leq[\mathrm{Fe} / \mathrm{H}] \leq-1$ metallicity range. For the weak $\mathrm{Fe}_{\mathrm{I}}$ line with the same excitation potential, $3 \mathrm{D}-1 \mathrm{D} \simeq-0.4,-0.3$, and $-0.17 \mathrm{dex}$ in the $[\mathrm{Fe} / \mathrm{H}]=-3,-2$, and -1 models, respectively. The 3D effects are overall weak for the $\mathrm{Fe}$ II lines arising from the ground state. For example, for the moderate strength line $(E W \simeq 50 \mathrm{~m} \AA)$, $3 \mathrm{D}-1 \mathrm{D}=-0.03,+0.15$, and $+0.15 \mathrm{dex}$ in the $[\mathrm{Fe} / \mathrm{H}]=-3,-2$, and -1 model, respectively. We assumed that 3D-1D corrections for the investigated Eu II lines are similar to that for the "fictitious" Fe II line with $E_{\text {exc }}=0 \mathrm{eV}$. The 3D-1D correction for $\mathrm{Pb}$ I $4057 \AA$ lies, probably, between those for Na I and Fe I. When it is as large as that for Na I and we account for the 3D effects, the $\mathrm{Pb} / \mathrm{Eu}$ abundance ratio is approximately 0.1 dex lower for the two r-II stars and 0.25 dex lower for both the low-metallicity and mildly metal-poor samples. With these changes, our conclusions remain valid. If the 3D-1D correction for $\mathrm{Pb}_{\text {I }} 4057 \AA$ is as large as that for $\mathrm{Fe} \mathrm{I}$, the mean $\mathrm{Pb} / \mathrm{Eu}$ abundance ratios of the r-II stars and the LMS stars are expected to be approximately 0.4 dex lower than the corresponding 1D+non-LTE values. However, in this case, it would be difficult to understand 


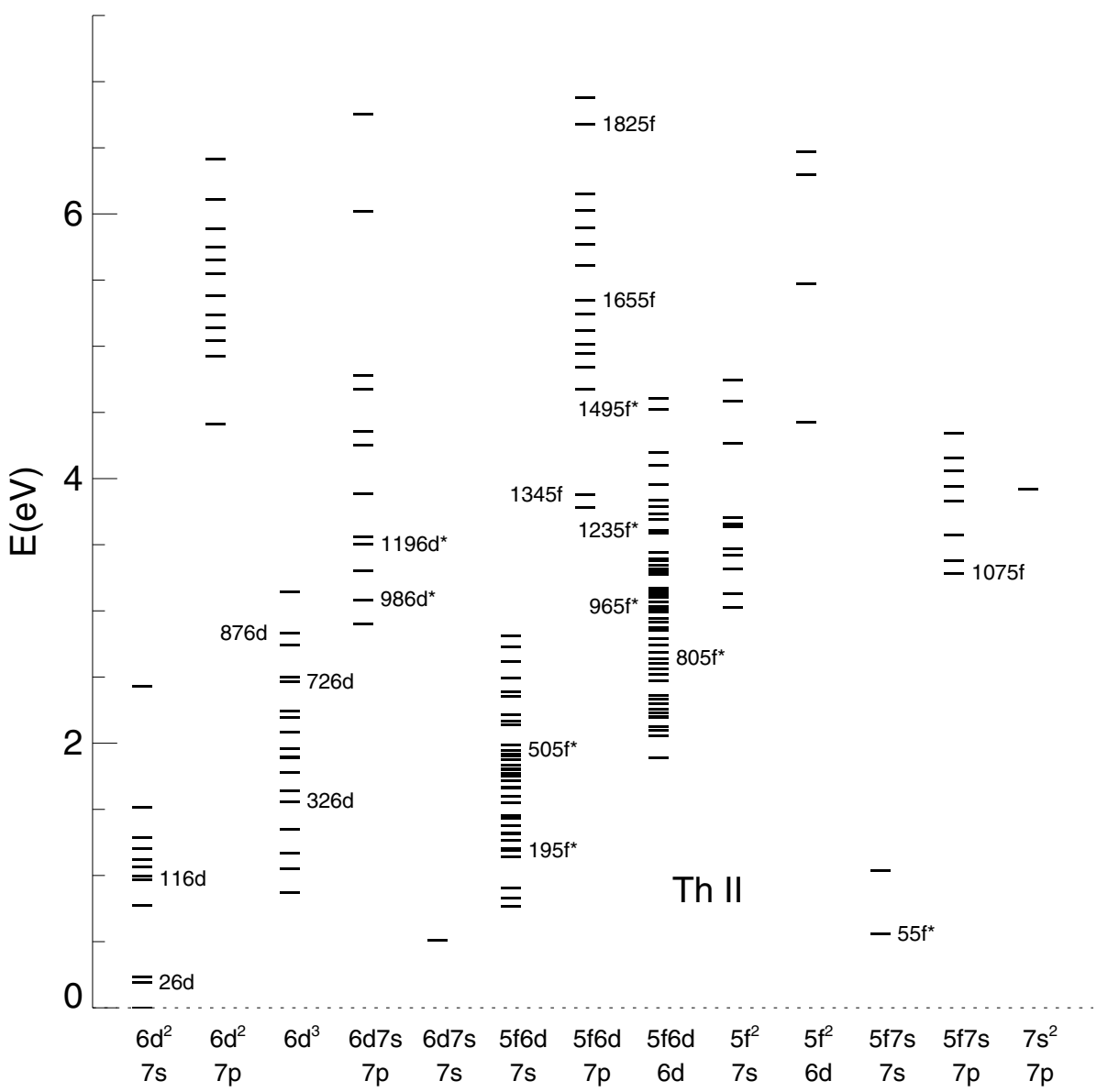

Fig. 6. Model atom of Th II. The names of the levels important for understanding the non-LTE mechanisms are indicated.

why the $\mathrm{Pb} / \mathrm{Eu}$ abundance ratio of the strongly r-process enhanced stars is significantly lower (by $0.5 \mathrm{dex}$ ) than the Solar System r-process value.

We note here that full 3D non-LTE computations have so far only been performed for $\mathrm{Li}$ I in the Sun and metal-poor stars (Asplund et al. 2003; Barklem et al. 2003; Cayrel et al. 2007; Sbordone et al. 2010) and for O I in the Sun (Asplund et al. 2004). It has been found that the 3D+non-LTE effects are small overall for the Li I resonance line because the line strengthening due to the cooler temperatures in the upper atmospheric layers of the 3D model on the one hand, and line weakening from increased overionization on the other hand, largely cancel each other out. For O I, the non-LTE abundance corrections were found to be similar in the 3D and 1D solar model atmospheres. In contrast, Shchukina et al. (2005) predicted stronger non-LTE effects for $\mathrm{Fe}_{\mathrm{I}}$ in the 3D than the 1D model, based on their 1.5D+non-LTE calculations for the Sun and HD 140283 (5700/3.7/-2.5). Full 3D non-LTE computations need to be done for $\mathrm{Pb}$ I to decide whether there is any difference in the $\mathrm{Pb} / \mathrm{Eu}$ abundance ratio between the 3D+non-LTE and 1D+non-LTE cases and how this might affect our conclusions.

\section{Non-LTE calculations for Th II}

\subsection{Thorium model atom}

Energy levels. In the range of stellar parameters that we consider here, the majority of the element exists as Th II. For example, the fraction of neutral thorium, with its ionization energy $\chi_{\text {ion }}=6.3 \mathrm{eV}$, does not exceed $10^{-3}$ throughout the $4500 / 1 /-3$ atmosphere and $6 \times 10^{-3}$ throughout the solar atmosphere. We thus neglected ThI when constructing the model atom. The Th II term structure is produced by multiple electronic configurations (see Fig. 6) and consists of thousands of energy levels. The ground configuration is $6 \mathrm{~d}^{2} 7 \mathrm{~s}$. We used the energy levels with $E_{\text {exc }} \leq 6.88 \mathrm{eV}$, which amount to 416 levels from Blaise \& Wyart (1992). The higher excitation levels play a minor role in the population and depopulation of Th II, because the next ionization stage, Th III, represents a minor fraction of the thorium abundance. For example, the contribution of Th III to the thorium abundance is smaller than $0.2 \% / 1.5 \%$ in the cool $\mathrm{VMP} /$ solar models outside $\log \tau_{5000}=0$. The levels with a common parity and close energies were combined whenever the energy separation was smaller than $0.01 \mathrm{eV}$ at $E_{\mathrm{exc}}<4 \mathrm{eV}$ and smaller than $0.1 \mathrm{eV}$ at higher $E_{\text {exc }}$. The final model atom includes 184 levels of Th II (Fig. 6).

The Th III ground state $5 \mathrm{f}^{3} \mathrm{~d}^{3} \mathrm{H}_{4}^{\circ}$ is a poor representation of the Th III ionization stage. The odd 5f6d and 5f7s and the even $6 \mathrm{~d}^{2}$ and $6 \mathrm{~d} 7 \mathrm{~s}$ configurations of Th III produce many lowexcitation levels. The energy of $6 \mathrm{~d}^{2}{ }^{3} \mathrm{~F}_{2}, E_{\mathrm{exc}}=63.2 \mathrm{~cm}^{-1}$, is the smallest distance between the lowest levels of both parities ever found in an atomic spectrum. As a result, the Th III partition function $(U)$ is significantly larger than the statistical weight $g=9$ of the ground state. For example, $U($ Th III $)=49.14$ at the temperature $T=4990 \mathrm{~K}$ and grows to $U($ Th III $)=81.11$ at $T=8318 \mathrm{~K}$. These values were computed using the Th III energy levels from Blaise \& Wyart (1992). Assuming that the low-excitation levels of Th III are thermally coupled to the ground state, we included Th III in the model atom as a single state with $g=49$, while keeping the energy of the ground state. 
Radiative rates. Accurate $g f$-values based on measured natural radiative lifetimes and branching ratios were obtained by Nilsson et al. (2002) for 180 transitions between the lowest $\left(E_{\mathrm{exc}}=0-2.25 \mathrm{eV}\right)$ even parity levels of the $6 \mathrm{~d}^{2} 7 \mathrm{~s}, 6 \mathrm{~d} 7 \mathrm{~s}^{2}$, and $6 \mathrm{~d}^{3}$ electronic configurations and the intermediate energy $\left(E_{\mathrm{exc}}=\right.$ $2.26-4.08 \mathrm{eV}$ ) odd parity levels. For another 1244 transitions, we used $g f$-values of Kurucz \& Bell (1995), which are accessible via the vald database (Kupka et al. 1999). These two sources provide the data for $955 b-b$ transitions between combined levels in our model atom. They were all included in SE calculations. We refer to this atomic model as Th- 1 .

The second atomic model, Th-2, includes the extended system of $b-b$ radiative transitions. We estimated the total number of allowed transitions in the system of 416 Th II levels assuming that every transition between the different parity levels with $\Delta J=0, \pm 1$ is an allowed one. The exceptions were the $J=0 \leftrightarrow J=0$ transitions. This gives 20214 transitions, and for 18790 of them, oscillator strengths $\left(f_{i j}\right)$ are missing. A total of 1562 of the latter transitions, with the energy separation of less than $0.25 \mathrm{eV}$, were treated as forbidden ones because of the insignificant contribution of the radiative to the total transition rate, irrespective of the transition $g f$-value. For everyone of the remaining 17228 transitions, $f_{i j}=10^{-4}$ was adopted. This choice was based on the inspection of the known $g f$-values for Th II. No transition from the Nilsson et al. (2002) and Kurucz \& Bell (1995) data sets has $f_{i j} \leq 10^{-4}$. In this atomic model, the $5661 b-b$ transitions were included in the SE calculations.

The photoionization cross-sections were computed using the hydrogen approximation formula with $n=n_{\text {eff }}$, because no accurate data were available for the Th in levels.

Collisional rates. The same formulae as for $\mathrm{Pb}$ I were employed for the transitions in Th II, assuming that $\Upsilon=2$ for the forbidden ones.

\subsection{Departures from LTE for Th II}

The non-LTE calculations for Th II were performed with the MARCS solar model atmosphere and the models representing the atmospheres of VMP stars with $[\mathrm{Fe} / \mathrm{H}]=-2$ and -3 (see Table 3 ). Figure 7 shows the departure coefficients of the selected Th II levels in the 5780/4.44/0 and 4500/1.0/-3 models. We found that the lowest 72 levels, i.e., up to the level denoted as $726 \mathrm{~d}\left(E_{\mathrm{exc}}=2.46 \mathrm{eV}\right)$, keep nearly TE populations $(b \simeq 1)$ in the atmosphere inside $\log \tau_{5000}=-2$ and -1 for the solar and VMP models, respectively. For all the higher excitation levels, $b>1$. This is because the population of the odd parity $5 \mathrm{f}^{2} \mathrm{~d}^{2}$ and $6 \mathrm{~d} 7 \mathrm{~s} 7 \mathrm{p}$ levels with $E_{\text {exc }}=3-4.8 \mathrm{eV}$ is controlled by the strong radiative transitions from the three lowest $6 \mathrm{~d}^{2} 7 \mathrm{~s}$ states and the $6 \mathrm{~d} 7 \mathrm{~s}^{2}$ level, such as $6 \mathrm{~d}^{2} 7 \mathrm{~s}^{4} \mathrm{~F}_{3 / 2}-6 \mathrm{~d} 7 \mathrm{~s} 7 \mathrm{p}\left(E_{\text {exc }}=3.084 \mathrm{eV}\right.$, $J=5 / 2$ ) denoted in our model atom as $16 \mathrm{~d}-986 \mathrm{~d} *$ that produces the line at $4019 \AA, 16 \mathrm{~d}-965 \mathrm{f} *$ (4094 $\AA$ ), 26d-1196d*(3741 $\AA$ ), and $36 \mathrm{~d}-1495 \mathrm{f} *(2895 \AA)$. These transitions are pumped by the ultraviolet $J_{v}-B_{v}\left(T_{\mathrm{e}}\right)$ excess radiation and produce enhanced excitation of the upper levels far within the atmosphere. This overpopulation is redistributed to the even-parity high-excitation levels through inelastic collisions.

The Th-2 atomic model includes more pumping transitions than the Th- 1 one, and the departures from LTE are expected to be stronger when using the Th-2 model. We checked the populations of the lower and upper levels of the Th II $4019 \AA$ transition. In the line formation layers, around $\log \tau_{5000}=-0.45$ and -0.55 in the solar and 4500/1.0/-3 models, respectively, the difference in the non-LTE populations between the Th- 2 and Th- 1 atomic
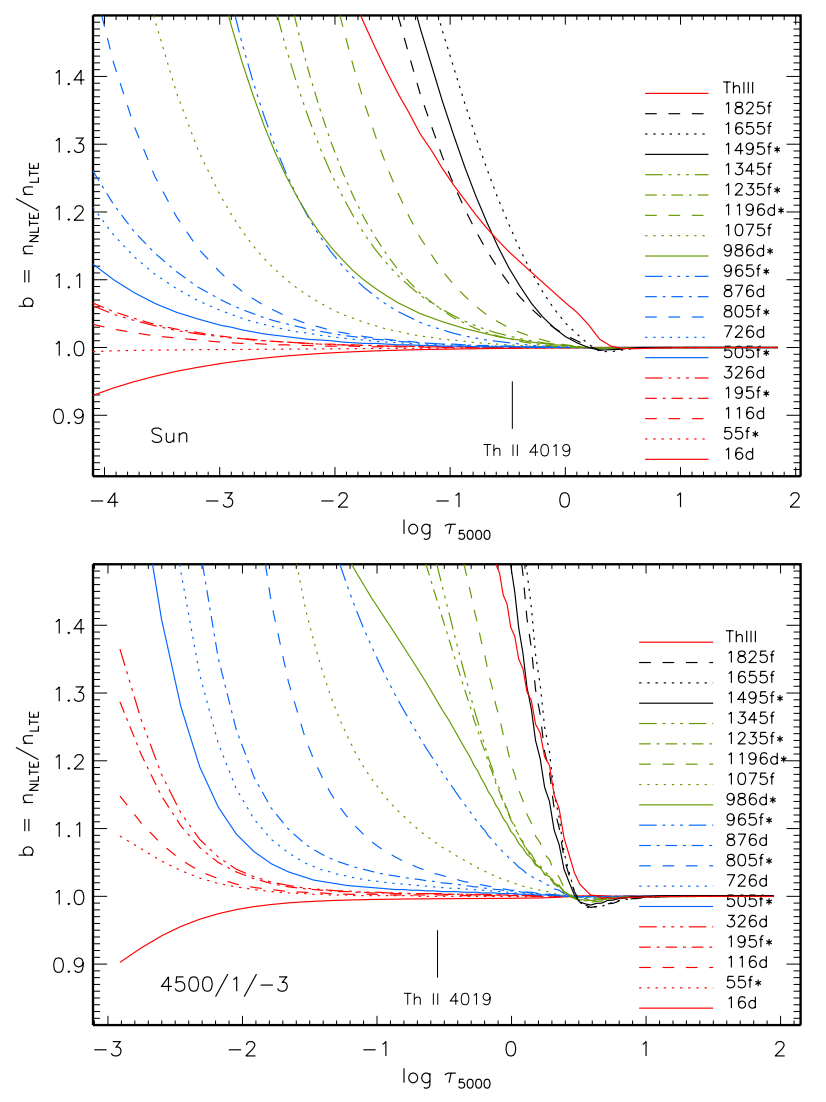

Fig. 7. The same as in Fig. 3 for the selected levels of Th II and the Th III ground state from the calculations with the Th- 2 atomic model. The vertical lines indicate the locations of line core formation depths for Th II 4019.

Table 3. Non-LTE abundance corrections (dex) for the Th II lines from the calculations with $S_{\mathrm{H}}=0.1$.

\begin{tabular}{cccccc}
\hline \hline$T_{\text {eff }}$ & $\log g$ & {$[\mathrm{Fe} / \mathrm{H}]$} & {$[\mathrm{Th} / \mathrm{Fe}]^{a}$} & \multicolumn{2}{c}{$\Delta_{\text {NLTE }}$} \\
\cline { 5 - 6 } & & & & $4019 \AA$ & $4086 \AA$ \\
\hline 5780 & 4.44 & 0 & $0.09^{b}$ & 0.01 & $-^{c}$ \\
4000 & 0.0 & -2 & 0.4 & 0.21 & 0.14 \\
4000 & 0.5 & -2 & 0.4 & 0.13 & 0.08 \\
4250 & 0.5 & -2 & 0.4 & 0.16 & 0.09 \\
4250 & 1.0 & -2 & 0.4 & 0.10 & 0.06 \\
4500 & 1.0 & -2 & 0.4 & 0.12 & 0.07 \\
4500 & 1.5 & -2 & 0.4 & 0.07 & 0.04 \\
4750 & 1.5 & -2 & 0.4 & 0.09 & 0.05 \\
4750 & 2.0 & -2 & 0.4 & 0.06 & 0.03 \\
5250 & 2.5 & -2 & 0.4 & 0.05 & - \\
4500 & 1.0 & -3 & 1.75 & 0.12 & 0.06 \\
4500 & 1.5 & -3 & 1.75 & 0.07 & 0.03 \\
4750 & 1.0 & -3 & 1.75 & 0.15 & 0.09 \\
4750 & 1.5 & -3 & 1.75 & 0.09 & 0.05 \\
\hline
\end{tabular}

Notes. (a) Thorium abundance used in non-LTE calculations. (b) Absolute thorium abundance, $\log \varepsilon_{\mathrm{Th}}{ }^{(c)}$ Calculated equivalent width is smaller than $1 \mathrm{~m} \AA$.

models is negligible for the Th II ground state. This is easy to understand because the lowest Th II levels contain the majority of the element, and no mechanism is able to significantly change their populations away from the properties of the TE ones. For the upper level, $986 \mathrm{~d}^{*}$, a small difference of $3 \%$ was found in 
the VMP model between using Th-2 and Th-1, and very similar populations were obtained in the solar model.

The Th II lines used in abundance analyses all arise from either the ground state or low-excitation levels with $E_{\text {exc }} \leq 0.51 \mathrm{eV}$, for which $b \simeq 1$ holds in the line formation layers (Fig. 7). Nevertheless, for each line, non-LTE leads to its weakening relative to the LTE strength, owing to the overpopulation of the upper level relative to the TE population that results in $b_{\mathrm{u}} / b_{1}>1$ and the increase in the line source function above the Planck function in the line-formation layers. Overall, the derived nonLTE abundance corrections are positive. For the Sun, the departures from LTE are small irrespective of the assumed $S_{\mathrm{H}}$ value, such that $\Delta_{\mathrm{NLTE}}($ Th II $4019 \AA)=0.06$ and 0.00 dex in the case of $S_{\mathrm{H}}=0$ and 1 , respectively. In the VMP models, the nonLTE correction is very sensitive to treatment of inelastic collisions with $\mathrm{H}_{\mathrm{I}}$ atoms. For example, for $4500 / 1 /-3$, it amounts to $\Delta_{\text {NLTE }}=+0.52$ dex in the calculations with pure electronic collisions and it varies between $\Delta_{\mathrm{NLTE}}=+0.12$ and +0.03 dex when collisions with $\mathrm{H}_{\mathrm{I}}$ atoms are taken into account and when $S_{\mathrm{H}}=$ 0.1 to 1 . The use of the Th-1 atomic model results in non-LTE corrections that are 0.01 dex smaller.

Table 3 presents $\Delta_{\text {NLTE }}$ for the two lines, Th II 4019 and $4086 \AA$, from the calculations with the Th- 2 atomic model and $S_{\mathrm{H}}=0.1$. For a given $T_{\text {eff }}$, the departures from LTE increase toward lower surface gravity owing to the decreasing contribution of the collisional to the total transition rates. Maximum values of $\Delta_{\text {NLTE }}$ were calculated for the $4000 / 0.0 /-2$ model, and amount to +0.21 dex for Th II $4019 \AA$ and +0.14 dex for Th II $4086 \AA$. The metallicity effect is less pronounced in Table 3 because, for the $[\mathrm{Fe} / \mathrm{H}]=-3$ models, the non-LTE calculations were performed with a higher $\mathrm{Th}$ abundance than in the $[\mathrm{Fe} / \mathrm{H}]=-2$ models.

\section{Conclusions}

We have developed a comprehensive model atom for neutral lead using atomic data for the energy levels and transition probabilities inferred from laboratory measurements and theoretical predictions. We have performed non-LTE calculations for $\mathrm{Pb}$ i for the first time for the Sun and for a set of stellar parameters characteristic of metal-poor stars in the $-2.95 \leq[\mathrm{Fe} / \mathrm{H}] \leq-0.59$ metallicity range. We found that the main non-LTE mechanism for $\mathrm{Pb}_{\mathrm{I}}$ is the ultraviolet overionization, although radiative pumping of the strong transitions arising from the low-lying levels tends to produce enhanced excitation of the $\mathrm{Pb}$ I levels with $E_{\text {exc }}>4 \mathrm{eV}$. Overall, non-LTE leads to weakened Pb I lines and positive non-LTE abundance corrections. We have found that the departures from LTE increase with decreasing metallicity and, for a given metallicity, increase toward higher effective temperatures and lower surface gravities. The main source of the uncertainty in the calculated non-LTE abundance corrections are poorly known inelastic collisions with $\mathrm{H}_{\mathrm{I}}$ atoms. From our analysis of either solar or stellar $\mathrm{Pb}$ i lines, we have been unable to empirically constrain their efficiency in SE calculations. With $S_{\mathrm{H}}=0.1$, chosen based on our estimates for $\mathrm{Ca}$ I-Ca II and Fe I-Fe II (Mashonkina et al. 2007, 2011), $\Delta_{\text {NLTE }}$ of Pb I $4057 \AA$ ranges between $0.16 \mathrm{dex}$ in the solar model and $0.56 \mathrm{dex}$ in the 4825/1.5/-2.9 model.

In addition, we have built a model atom for singly-ionized thorium using atomic data for the energy levels derived from laboratory measurements. Our non-LTE calculations for Th II are again the first that have been performed for the Sun and the small grid of model atmospheres with $[\mathrm{Fe} / \mathrm{H}]=-2$ and -3 . In contrast to $\mathrm{Pb}$, Th II is the majority species in the stellar parameter range that we have covered, and the main non-LTE mechanism for Th II is connected to the pumping transitions arising from the low-excitation levels, with $E_{\text {exc }}<1 \mathrm{eV}$. Overall, non-LTE leads to weakened Th II lines and positive non-LTE abundance corrections. Compared with $\mathrm{Pb}$ I, the departures from LTE for Th II are even more sensitive to the efficiency of collisions with $\mathrm{H}_{\mathrm{I}}$ atoms. With $S_{\mathrm{H}}=0.1, \Delta_{\mathrm{NLTE}}$ of the investigated Th II lines nowhere exceeds 0.21 dex.

We caution against using the LTE assumption in stellar analyses of both the $\mathrm{Pb}$ abundance and the elemental abundance ratios involving $\mathrm{Pb}$ and some other element observed in the lines of their majority species, such as Eu, Th, or U.

Taking advantage of our $\mathrm{SE}$ approach for $\mathrm{Pb}$ I, we have found good agreement, to within 0.03 dex, between meteoritic and absolute solar abundances from the calculations with the Holweger $\&$ Müller (1974) solar model atmosphere and $S_{\mathrm{H}}=0.1$. Our final value is $\log \varepsilon_{\mathrm{Pb}, \odot}=2.09$.

We then calculated the non-LTE abundances of $\mathrm{Pb}$ and $\mathrm{Eu}$ for the Roederer et al. (2010) metal-poor stars and complemented that sample with data for HE 1523-0901 (Frebel et al. 2007) and HD 29907 (Sitnova \& Mashonkina 2011). We found that the two strongly r-process enhanced (r-II) stars have very similar $\mathrm{Pb} / \mathrm{Eu}$ abundance ratios, with the mean $\log \varepsilon_{\mathrm{NLTE}}(\mathrm{Pb} / \mathrm{Eu})=$ $0.68 \pm 0.01$, and that the waiting-point r-process model presented by Roederer et al. (2009) reproduces the observations very well. The revised $\mathrm{Pb} / \mathrm{Eu}$ abundance ratios of the $\mathrm{r}$-II stars match, within the error bars, the corresponding solar r-process ratio. Thus, the universality of the r-process has been proven not only for the second r-process peak elements from $\mathrm{Ba}$ to $\mathrm{Hf}$ as found earlier by Sneden et al. (1996); Hill et al. (2002), and Sneden et al. (2008, and references therein), but also for the heavier element $\mathrm{Pb}$.

It has been found that the stars in the $-2.3 \leq[\mathrm{Fe} / \mathrm{H}]<$ -0.6 metallicity range, reveal a clear upward trend in the $\log \varepsilon(\mathrm{Pb} / \mathrm{Eu})-[\mathrm{Fe} / \mathrm{H}]$ plane. Following Roederer et al. (2010), we separated these stars into two groups depending on their metallicity. The lower metallicity stars $([\mathrm{Fe} / \mathrm{H}]<-1.4)$ turned out to have, on average, 0.51 dex higher $\mathrm{Pb} / \mathrm{Eu}$ ratios than the r-II stars. This led us to conclude that the s-process production of lead started before the Galactic metallicity had increased to $[\mathrm{Fe} / \mathrm{H}]=-2.3$. We note that the studies of Burris et al. (2000) and Simmerer et al. (2004), who measured the (Ba, La, Nd)/(Eu, Dy) abundance ratios of metal-poor stars, also found that the s-process was probably active as early as the times at which $[\mathrm{Fe} / \mathrm{H}]=-2.3$ and -2.6 , respectively.

The mean ratio, $\log \varepsilon_{\mathrm{NLTE}}(\mathrm{Pb} / \mathrm{Eu})=1.58 \pm 0.31$, for the mildly metal-poor subsample $(-1.4 \leq[\mathrm{Fe} / \mathrm{H}] \leq-0.59)$ turned out to be very close to the Solar System value, $\log \varepsilon_{\text {met }}(\mathrm{Pb} / \mathrm{Eu})=$ 1.53 (Lodders et al. 2009). This agrees with the theoretical result of Travaglio et al. (2001), who predicted that the AGB stars with $[\mathrm{Fe} / \mathrm{H}] \simeq-1$ made the greatest contribution to the abundance of s-nuclei of lead in the presolar nebula from which the Sun originated.

Acknowledgements. L.M. is supported by the Swiss National Science Foundation (SCOPES project No. IZ73Z0-128180/1) and the Russian Leading Scientific School grant 3602.2012.2. A.F. is, in part, supported by a Clay Fellowship administered by the Smithsonian Astrophysical Observatory. We used the MARCS model atmosphere library and the NIST and VALD databases.

\section{References}

Ahad, A., Nadeem, A., Bhatti, S. A., \& Baig, M. A. 2005, Eur. Phys. J. D, 32, 271

Anders, E., \& Grevesse, N. 1989, Geochim. Cosmochim. Acta, 53, 197 
Asplund, M., Carlsson, M., \& Botnen, A. V. 2003, A\&A, 399, L31

Asplund, M., Grevesse, N., Sauval, A. J., Allende Prieto, C., \& Kiselman, D. 2004, A\&A, 417, 751

Asplund, M., Grevesse, N., Sauval, A. J., \& Scott, P. 2009, ARA\&A, 47, 481

Barklem, P. S., Belyaev, A. K., \& Asplund, M. 2003, A\&A, 409, L1

Barklem, P. S., Belyaev, A. K., Guitou, M., et al. 2011, A\&A, 530, A94

Biémont, E., Garnir, H. P., Palmeri, P., Li, Z. S., \& Svanberg, S. 2000, MNRAS, 312, 116

Blaise, J., \& Wyart, J. F. 1992, Energy levels and atomic spectra of actinides, International tables of selected constants (The French Centre National de la Recherche Scientifique and Belgian Government)

Brault, J., Testerman, L., \& Observatory, K. P. N. 1972, Kitt Peak Solar Atlas: 2942-10800 A (Kitt Peak National Observatory)

Brown, C. M., Tilford, S. G., \& Ginter, M. L. 1977, J. Opt. Soc. Am. (1917-1983), 67, 1240

Burris, D. L., Pilachowski, C. A., Armandroff, T. E., et al. 2000, ApJ, 544, 302

Butler, K., \& Giddings, J. 1985, Newsletter on the analysis of astronomical spectra, No. 9, University of London

Cayrel, R., Hill, V., Beers, T. C., et al. 2001, Nature, 409, 691

Cayrel, R., Steffen, M., Chand, H., et al. 2007, A\&A, 473, L37

Christlieb, N., Beers, T. C., Barklem, P. S., et al. 2004, A\&A, 428, 1027

Collet, R., Asplund, M., \& Trampedach, R. 2007, A\&A, 469, 687

Cowan, J. J., \& Thielemann, F.-K. 2004, Physics Today, 57, 47

Cowan, R. D. 1981, The Theory of Atomic Structure and Spectra (Berkeley, California, USA: Univ. of California Press)

Dembczyński, J., Stachowska, E., Wilson, M., Buch, P., \& Ertmer, W. 1994, Phys. Rev. A, 49, 745

Drawin, H.-W. 1961, Z. Phys., 164, 513

Frebel, A., Christlieb, N., Norris, J. E., et al. 2007, ApJ, 660, L117

Fuhr, J. R., \& Wiese, W. L. 1992, NIST Atomic Transition Probability Tables,

CRC Handbook of Chemistry \& Physics, 77th edn., ed. D. R. Lide (Boca Raton, FL: CRC Press, Inc.)

Gallino, R., Arlandini, C., Busso, M., et al. 1998, ApJ, 497, 388

Gustafsson, B., Edvardsson, B., Eriksson, K., et al. 2008, A\&A, 486, 951

Hasegawa, S., \& Suzuki, A. 1996, Phys. Rev. A, 53, 3014

Hayek, W., Asplund, M., Collet, R., \& Nordlund, ̊. 2011, A\&A, 529, A158

Heppinstall, R., \& Marr, G. V. 1969, Proc. Roy. Soc. A, 310, 35

Hill, V., Plez, B., Cayrel, R., et al. 2002, A\&A, 387, 560

Hillebrandt, W. 1978, Space Sci. Rev., 21, 639

Holweger, H., \& Müller, E. A. 1974, Sol. Phys., 39, 19

Ivans, I. I., Sneden, C., Gallino, R., Cowan, J. J., \& Preston, G. W. 2005, ApJ, 627, L145

Kratz, K.-L., Farouqi, K., Pfeiffer, B., et al. 2007, ApJ, 662, 39
Krause, M. O., Gerard, P., \& Fahlman, A. 1986, Phys. Rev. A, 34, 4511

Kupka, F., Piskunov, N., Ryabchikova, T. A., Stempels, H. C., \& Weiss, W. W. 1999, A\&AS, 138, 119

Kurucz, R. L. 1994, SYNTHE Spectrum Synthesis Programs and Line Data, CD-ROM No. 18 (Cambridge, Mass.)

Kurucz, R. L., \& Bell, B. 1995, Atomic Line Data. CD-ROM No. 23, http:// kurucz.harvard.edu

Li, Z. S., Zhankui, J., Berzinsh, U., Persson, A., \& Svanberg, S. 2001, J. Phys. B At. Mol. Phys., 34, 3501

Lodders, K., Plame, H., \& Gail, H.-P. 2009, in Landolt-Börnstein - Group VI Astronomy and Astrophysics Numerical Data and Functional Relationships in Science and Technology, Vol. 4B: Solar System, 4.4, ed. J. E. Trümper, 44 Lugaro, M., Karakas, A. I., Stancliffe, R. J., \& Rijs, C. 2012, ApJ, 747, 2

Mashonkina, L., \& Gehren, T. 2000, A\&A, 364, 249

Mashonkina, L., Korn, A. J., \& Przybilla, N. 2007, A\&A, 461, 261

Mashonkina, L., Gehren, T., Shi, J.-R., Korn, A. J., \& Grupp, F. 2011, A\&A, 528, A87

Mihalas, D. 1978, Stellar atmospheres, 2nd edn. (San Francisco: W. H. Freeman and Co.)

Moore, C. E. 1958, Atomic Energy Levels, Natl. Bur. Stand. (US) Circ. 467, Vol. III

Müller, M., Böwering, N., Schäfers, F., \& Heinzmann, U. 1990, Phys. Scr., 41, 38

Nilsson, H., Zhang, Z. G., Lundberg, H., Johansson, S., \& Nordström, B. 2002, A\&A, 382, 368

Plez, B., Hill, V., Cayrel, R., et al. 2004, A\&A, 428, L9

Reetz, J. K. 1991, Diploma Thesis, Universität München

Roederer, I. U., Kratz, K.-L., Frebel, A., et al. 2009, ApJ, 698, 1963

Roederer, I. U., Cowan, J. J., Karakas, A. I., et al. 2010, ApJ, 724, 975

Rybicki, G. B., \& Hummer, D. G. 1991, A\&A, 245, 171

Rybicki, G. B., \& Hummer, D. G. 1992, A\&A, 262, 209

Sbordone, L., Bonifacio, P., Caffau, E., et al. 2010, A\&A, 522, A26

Shchukina, N. G., Trujillo Bueno, J., \& Asplund, M. 2005, ApJ, 618, 939

Simmerer, J., Sneden, C., Cowan, J. J., et al. 2004, ApJ, 617, 1091

Sitnova, T. M., \& Mashonkina, L. I. 2011, Astron. Lett., 37, 480

Sneden, C., McWilliam, A., Preston, G. W., et al. 1996, ApJ, 467, 819

Sneden, C., Cowan, J. J., \& Gallino, R. 2008, ARA\&A, 46, 241

Steenbock, W., \& Holweger, H. 1984, A\&A, 130, 319

Takeda, Y. 1995, PASJ, 47, 463

Travaglio, C., Galli, D., Gallino, R., et al. 1999, ApJ, 521, 691

Travaglio, C., Gallino, R., Busso, M., \& Gratton, R. 2001, ApJ, 549, 346

van Regemorter, H. 1962, ApJ, 136, 906

Wood, D. R., \& Andrew, K. L. 1968, J. Opt. Soc. Am., 58, 818 